Preprint typeset in JHEP style - HYPER VERSION

hep-th/0611266

BONN-TH-2006-07

IFT UWr 0211/006

\title{
Recursion representation of the Neveu-Schwarz superconformal block
}

\author{
Leszek Hadasz* \\ Physikalisches Institut, Rheinische Friedrich-Wilhelms-Universität, Nußallee 12, \\ 53115 Bonn, Germany \\ and \\ M. Smoluchowski Institute of Physics, Jagiellonian University, Reymonta 4, \\ 30-059 Kraków, Poland \\ Zbigniew Jaskólski ${ }^{\dagger}$ \\ Institute of Theoretical Physics, University of Wroctaw, pl. M. Borna, \\ 950-204 Wrocław, Poland

\section{Paulina Suchanek ${ }^{\ddagger}$ \\ M. Smoluchowski Institute of Physics, Jagiellonian University, Reymonta 4, 30-059 Kraków, Poland}

\begin{abstract}
Four-point super-conformal blocks for the $\mathrm{N}=1$ Neveu-Schwarz algebra are defined in terms of power series of the even super-projective invariant. Coefficients of these expansions are represented both as sums over poles in the "intermediate" conformal weight and as sums over poles in the central charge of the algebra. The residua of these poles are calculated in both cases. Closed recurrence relations for the block coefficients are derived.
\end{abstract}

KEYwords: N=1 NS algebra, conformal block.

\footnotetext{
*Alexander von Humboldt Fellow; e-mail: hadasz@th.if.uj.edu.pl

†jask@ift.uni.wroc.pl

${ }^{\ddagger}$ suchanek@th.if.uj.edu.pl
} 


\section{Contents}

1. Introduction 1

2. Four-point correlation functions of the Neveu-Schwarz sector 2

3. NS supermodule

4. NS chiral vertex operator 6

5. NS superconformal blocks 9

6. Recurrence relations 12

\section{Introduction}

Since the appearance of the seminal BPZ work [1], the conformal block has been recognized as one of the basic objects in conformal field theory. In spite of the progress achieved over the years its explicit calculation is still one of the most difficult problems in CFT. Not only the form of a general conformal block is unknown, but also its analytic properties still have a status of conjectures rather than theorems. On the other hand, from the practical point of view there exist very efficient recursive methods of an approximate (with arbitrary precision), analytic determination of a general 4-point conformal block [2-4]. They were used for instance in checking the conformal bootstrap in the Liouville theory with the DOZZ coupling constants [5], in study of the $c \rightarrow 1$ limit of minimal models [6] or in obtaining new results in the classical geometry of hyperbolic surfaces [7]. In a more general context of an arbitrary CFT model these methods allow for efficient numerical calculations of any 4-point function once the structure constants are known.

Similar problems can be also addressed in the supersymmetric conformal field theories (SCFT). It seems therefore desirable to develop analogous recursive methods also in this more complicated case. The present paper is aimed as a step in this direction. After defining conformal blocks for the Neveu-Schwarz algebra of the $N=1$ SCFT we give their representations in the form of a sum over poles in the central charge and in the "intermediate" conformal weight and derive a recurrence relation for residua of these poles. In the case of the sum over the poles in the central charge one can easily calculate the leading $(c \rightarrow \infty)$ term. This yields efficient recursion relations for the coefficients of the so called $x$-expansion of NS superconformal blocks. 
There are two problems which are natural continuations of the present work. The first one is to extend our constructions and results to the Ramond sector. The second is to develop recursion relations for the coefficients of the so called $q$-expansion [3, 4]. The latter problem requires an explicit calculations of the asymptotic behavior of the superconformal block for large intermediate weight. We hope to present the solutions to these problems in the near future.

\section{Four-point correlation functions of the Neveu-Schwarz sector}

The superconformal symmetry is generated by a pair of holomorphic currents $T(z), S(z)$ and their anti-holomorphic counterparts $\bar{T}(\bar{z}), \bar{S}(\bar{z})$, where $T$ and $\bar{T}$ are components of the energy-momentum tensor while $S$ and $\bar{S}$ have dimensions $(3 / 2,0)$ and $(0,3 / 2)$, respectively. The algebra of the modes of $T(z)$ and $S(z)$ is determined by the OPE-s

$$
\begin{aligned}
& T(z) T(0)=\frac{c}{2 z^{4}}+\frac{2}{z^{2}} T(z)+\frac{1}{z} \partial T(0)+\ldots, \\
& T(z) S(0)=\frac{3}{2 z^{2}} S(0)+\frac{1}{z} \partial S(0)+\ldots, \\
& S(z) S(0)=\frac{2 c}{3 z^{3}}+\frac{2}{z} T(0)+\ldots
\end{aligned}
$$

The space of fields of superconformal field theory (hereafter SCFT) decomposes onto the space of fields $\phi_{\mathrm{NS}}$ local with respect to $S(z)$, and the space of the Ramond fields $\phi_{\mathrm{R}}$ with the property that the correlation functions

$$
\left\langle S(z) \phi_{\mathrm{R}}\left(z_{1}, \bar{z}_{1}\right) \ldots\right\rangle
$$

(with the dots denoting any other operator insertions) change the sing upon analytic continuation in $z$ around the point $z=z_{1}$. It the present paper we shall discuss only the NS fields.

The locality properties of the NS field allow to write its OPE with $S(z)$ in the form

$$
S(z) \phi_{\mathrm{NS}}(0,0)=\sum_{k \in \mathbb{Z}+\frac{1}{2}} z^{k-\frac{3}{2}} S_{-k} \phi_{\mathrm{NS}}(0,0) .
$$

Together with the usual Virasoro generators $L_{n}$ defined by the OPE

$$
T(z) \phi_{\mathrm{NS}}(0,0)=\sum_{k \in \mathbb{Z}} z^{n-2} L_{-n} \phi_{\mathrm{NS}}(0,0),
$$

$S_{k}$ form the Neveu-Schwarz algebra determined by (2.1),

$$
\begin{aligned}
& {\left[L_{m}, L_{n}\right]=(m-n) L_{m+n}+\frac{c}{12} m\left(m^{2}-1\right) \delta_{m+n},} \\
& {\left[L_{m}, S_{k}\right]=\frac{m-2 k}{2} S_{m+k},} \\
& \left\{S_{k}, S_{l}\right\}=2 L_{k+l}+\frac{c}{3}\left(k^{2}-\frac{1}{4}\right) \delta_{k+l} .
\end{aligned}
$$


In the space of all NS fields there exist "super-primary" fields $\varphi_{\Delta, \bar{\Delta}}(z, \bar{z})$ with the conformal weights $\Delta, \bar{\Delta}$, which satisfy

$$
\begin{aligned}
{\left[L_{n}, \varphi_{\Delta, \bar{\Delta}}(0,0)\right] } & =\left[S_{k}, \varphi_{\Delta, \bar{\Delta}}(0,0)\right]=0, \quad n, k>0, \\
{\left[L_{0}, \varphi_{\Delta, \bar{\Delta}}(0,0)\right] } & =\Delta \varphi_{\Delta, \bar{\Delta}}(0,0),
\end{aligned}
$$

and similarly for the "right" generators $\bar{L}_{n}$ and $\bar{S}_{k}$. Each super-primary field is the "lowest" component of the superfield

$$
\Phi_{\Delta, \bar{\Delta}}(z, \theta ; \bar{z}, \bar{\theta})=\varphi_{\Delta, \bar{\Delta}}(z, \bar{z})+\theta \psi_{\Delta, \bar{\Delta}}(z, \bar{z})+\bar{\theta} \bar{\psi}_{\Delta, \bar{\Delta}}(z, \bar{z})-\theta \bar{\theta} \widetilde{\varphi}_{\Delta, \bar{\Delta}}(z, \bar{z}),
$$

where

$$
\psi_{\Delta, \bar{\Delta}}=\left[S_{-1 / 2}, \varphi_{\Delta, \bar{\Delta}}\right], \quad \bar{\psi}_{\Delta, \bar{\Delta}}=\left[\bar{S}_{-1 / 2}, \varphi_{\Delta, \bar{\Delta}}\right], \quad \widetilde{\varphi}_{\Delta, \bar{\Delta}}=\left\{S_{-1 / 2},\left[\bar{S}_{-1 / 2}, \varphi_{\Delta, \bar{\Delta}}\right]\right\},
$$

and $\theta, \bar{\theta}$ are Grassman (odd) numbers. The superfield (2.4) satisfies the equations:

$$
\begin{aligned}
& {\left[L_{n}, \Phi_{\Delta, \bar{\Delta}}(z, \theta ; \bar{z}, \bar{\theta})\right]=z^{n}\left[z \partial_{z}+(n+1)\left(\Delta+\frac{1}{2} \theta \partial_{\theta}\right)\right] \Phi_{\Delta, \bar{\Delta}}(z, \theta ; \bar{z}, \bar{\theta}),} \\
& {\left[\bar{L}_{n}, \Phi_{\Delta, \bar{\Delta}}(z, \theta ; \bar{z}, \bar{\theta})\right]=\bar{z}^{n}\left[\bar{z} \partial_{\bar{z}}+(n+1)\left(\bar{\Delta}+\frac{1}{2} \bar{\theta} \partial_{\bar{\theta}}\right)\right] \Phi_{\Delta, \bar{\Delta}}(z, \theta, \bar{z} ; \bar{\theta}),} \\
& {\left[S_{k}, \Phi_{\Delta, \bar{\Delta}}(z, \theta ; \bar{z}, \bar{\theta})\right]=z^{k-\frac{1}{2}}\left[z \partial_{\theta}-\theta z \partial_{z}-\theta(2 k+1) \Delta\right] \Phi_{\Delta, \bar{\Delta}}(z, \theta ; \bar{z}, \bar{\theta}),} \\
& {\left[\bar{S}_{k}, \Phi_{\Delta, \bar{\Delta}}(z, \theta ; \bar{z}, \bar{\theta})\right]=\bar{z}^{k-\frac{1}{2}}\left[\bar{z} \partial_{\bar{\theta}}-\bar{\theta} \bar{z} \partial_{\bar{z}}-\bar{\theta}(2 k+1) \bar{\Delta}\right] \Phi_{\Delta, \bar{\Delta}}(z, \theta ; \bar{z}, \bar{\theta}) .}
\end{aligned}
$$

Operators $L_{0}, S_{ \pm \frac{1}{2}}, L_{ \pm 1}$ (as well as their right counterparts) form a closed subalgebra of the NS algebra and exponentiate to the group of the "super-projective" transformations. These transformations allow to express three-point function of the primary superfields in the form

$$
\begin{aligned}
& \left\langle\Phi_{3}\left(z_{3}, \theta_{3} ; \bar{z}_{3}, \bar{\theta}_{3}\right) \Phi_{2}\left(z_{2}, \theta_{2} ; \bar{z}_{2}, \bar{\theta}_{2}\right) \Phi_{1}\left(z_{1}, \theta_{1} ; \bar{z}_{1}, \bar{\theta}_{1}\right)\right\rangle \\
& =Z_{32}^{\gamma_{1}} \bar{Z}_{32}^{\bar{\gamma}_{1}} Z_{31}^{\gamma_{2}} \bar{Z}_{31}^{\bar{\gamma}_{2}} Z_{21}^{\gamma_{3}} \bar{Z}_{21}^{\bar{\gamma}_{3}}\left\langle\Phi_{1}(\infty, 0 ; \infty, 0) \Phi_{2}(1, \Theta ; 1, \bar{\Theta}) \Phi_{3}(0,0 ; 0,0)\right\rangle
\end{aligned}
$$

where $\gamma_{1}=\Delta_{1}-\Delta_{2}-\Delta_{3}, \quad Z_{12}=z_{1}-z_{2}-\theta_{1} \theta_{2} \equiv z_{12}-\theta_{1} \theta_{2}$ etc.,

$$
\Theta=\frac{1}{\sqrt{z_{12} z_{13} z_{23}}}\left(\theta_{1} z_{23}+\theta_{2} z_{31}+\theta_{3} z_{12}-\frac{1}{2} \theta_{1} \theta_{2} \theta_{2}\right)
$$

and

$$
\Phi_{3}(\infty, 0 ; \infty, 0) \equiv \lim _{R \rightarrow \infty} R^{2 \Delta_{3}+2 \bar{\Delta}_{3}} \Phi_{3}(R, 0 ; R, 0)
$$

Thus, in contrast to the non-supersymmetric case, the three point function is determined by the superconformal symmetry up to two independent constants,

$$
\begin{aligned}
& C_{321}=\left\langle\varphi_{3}(\infty, \infty) \varphi_{2}(1,1) \varphi_{1}(0,0)\right\rangle \\
& \widetilde{C}_{321}=\left\langle\varphi_{3}(\infty, \infty) \widetilde{\varphi}_{2}(1,1) \varphi_{1}(0,0)\right\rangle .
\end{aligned}
$$


The super-projective invariance also allows to express a general four-point function through the four-point function of the form

$$
\left\langle\Delta_{4}, \bar{\Delta}_{4}\left|\Phi_{3}\left(1, \theta_{3} ; 1, \bar{\theta}_{3}\right) \Phi_{2}\left(z, \theta_{2} ; \bar{z}, \bar{\theta}_{2}\right)\right| \Delta_{1}, \bar{\Delta}_{1}\right\rangle
$$

Expanding the superfields in the fermionic arguments we get with the help of (2.4):

$$
\begin{aligned}
& \left\langle\Delta_{4}, \bar{\Delta}_{4}\left|\Phi_{3}\left(1, \theta_{3} ; 1, \bar{\theta}_{3}\right) \Phi_{2}\left(z, \theta_{2} ; \bar{z}, \bar{\theta}_{2}\right)\right| \Delta_{1}, \bar{\Delta}_{1}\right\rangle= \\
& \quad=\left\langle\Delta_{4}, \bar{\Delta}_{4}\left|\phi_{3}(1,1) \phi_{2}(z, \bar{z})\right| \Delta_{1}, \bar{\Delta}_{1}\right\rangle+\theta_{3} \bar{\theta}_{3} \theta_{2} \bar{\theta}_{2}\left\langle\Delta_{4}, \bar{\Delta}_{4}\left|\tilde{\phi}_{3}(1,1) \tilde{\phi}_{2}(z, \bar{z})\right| \Delta_{1}, \bar{\Delta}_{1}\right\rangle \\
& \quad-\theta_{2} \bar{\theta}_{2}\left\langle\Delta_{4}, \bar{\Delta}_{4}\left|\phi_{3}(1,1) \tilde{\phi}_{2}(z, \bar{z})\right| \Delta_{1}, \bar{\Delta}_{1}\right\rangle-\theta_{3} \bar{\theta}_{3}\left\langle\Delta_{4}, \bar{\Delta}_{4}\left|\tilde{\phi}_{3}(1,1) \phi_{2}(z, \bar{z})\right| \Delta_{1}, \bar{\Delta}_{1}\right\rangle \\
& \quad+\theta_{3} \theta_{2}\left\langle\Delta_{4}, \bar{\Delta}_{4}\left|\psi_{3}(1,1) \psi_{2}(z, \bar{z})\right| \Delta_{1}, \bar{\Delta}_{1}\right\rangle+\bar{\theta}_{3} \bar{\theta}_{2}\left\langle\Delta_{4}, \bar{\Delta}_{4}\left|\bar{\psi}_{3}(1,1) \bar{\psi}_{2}(z, \bar{z})\right| \Delta_{1}, \bar{\Delta}_{1}\right\rangle \\
& \quad+\theta_{3} \bar{\theta}_{2}\left\langle\Delta_{4}, \bar{\Delta}_{4}\left|\psi_{3}(1,1) \bar{\psi}_{2}(z, \bar{z})\right| \Delta_{1}, \bar{\Delta}_{1}\right\rangle+\bar{\theta}_{3} \theta_{2}\left\langle\Delta_{4}, \bar{\Delta}_{4}\left|\bar{\psi}_{3}(1,1) \psi_{2}(z, \bar{z})\right| \Delta_{1}, \bar{\Delta}_{1}\right\rangle .
\end{aligned}
$$

\section{NS supermodule}

Let $\nu_{\Delta}$ be the highest weight state with respect to the NS superconformal algebra (2.2)

$$
L_{0} \nu_{\Delta}=\Delta \nu_{\Delta}, \quad L_{m} \nu_{\Delta}=S_{k} \nu_{\Delta}=0, \quad m \in \mathbb{N}, k \in \mathbb{N}-\frac{1}{2},
$$

where $\mathbb{N}$ is the set of positive integers. We denote by $\mathcal{V}_{\Delta}^{f}$ the free vector space generated by all vectors of the form

$$
\nu_{\Delta, K M}=S_{-K} L_{-M} \nu_{\Delta} \equiv S_{-k_{i}} \ldots S_{-k_{1}} L_{-m_{j}} \ldots L_{-m_{1}} \nu_{\Delta}
$$

where $K=\left\{k_{1}, k_{2}, \ldots, k_{i}\right\} \subset \mathbb{N}-\frac{1}{2}$ and $M=\left\{m_{1}, m_{2}, \ldots, m_{j}\right\} \subset \mathbb{N}$ are arbitrary ordered sets of indices

$$
k_{i}<\ldots<k_{2}<k_{1}, \quad m_{j} \leq \ldots \leq m_{2} \leq m_{1}
$$

such that $|K|+|M|=k_{1}+\ldots+k_{i}+m_{1}+\ldots+m_{j}=f$.

The $\frac{1}{2} \mathbb{Z}$-graded representation of the NS superconformal algebra determined on the space

$$
\mathcal{V}_{\Delta}=\bigoplus_{f \in \frac{1}{2} \mathbb{N} \cup\{0\}} \mathcal{V}_{\Delta}^{f}, \quad \mathcal{V}_{\Delta}^{0}=\mathbb{C} \nu_{\Delta},
$$

by the relations (2.2) and (3.1) is called the NS supermodule of the highest weight $\Delta$ and the central charge $c$ (to avoid making the notation overloaded we omit the subscript $c$ at $\mathcal{V})$. Each $\mathcal{V}_{\Delta}^{f}$ is an eigenspace of $L_{0}$ with the eigenvalue $\Delta+f$. The space $\mathcal{V}_{\Delta}$ has also a natural $\mathbb{Z}_{2}$-grading:

$$
\mathcal{V}_{\Delta}=\mathcal{V}_{\Delta}^{+} \oplus \mathcal{V}_{\Delta}^{-}, \quad \mathcal{V}_{\Delta}^{+}=\bigoplus_{m \in \mathbb{N} \cup\{0\}} \mathcal{V}_{\Delta}^{m}, \quad \mathcal{V}_{\Delta}^{-}=\bigoplus_{k \in \mathbb{N}-\frac{1}{2}} \mathcal{V}_{\Delta}^{k},
$$

where $\mathcal{V}_{\Delta}^{ \pm}$are eigenspaces of the parity operator $(-1)^{F}=(-1)^{2\left(L_{0}-\Delta\right)}$. 
The tensor product $\mathcal{V}_{\Delta} \otimes \overline{\mathcal{V}}_{\bar{\Delta}}$ of the left and the right NS supermodules is defined as a graded tensor product of representations of $\mathbb{Z}_{2}$-graded algebras. The composition of tensor products of homogeneous elements is given by

$$
(A \otimes \bar{A})(B \otimes \bar{B})=(-1)^{\operatorname{deg}(\bar{A}) \cdot \operatorname{deg}(B)} A B \otimes \bar{A} \bar{B} .
$$

A nonzero element $\chi \in \mathcal{V}_{\Delta}^{f}$ of degree $f$ is called a singular vector if it satisfies the highest weight conditions (3.1) with $L_{0} \chi=(\Delta+f) \chi$. It generates its own NS supermodule $\mathcal{V}_{\Delta+f}$ which is a submodule of $\mathcal{V}_{\Delta}$.

The analysis of singular vectors can be facilitated by introducing a symmetric bilinear form $\langle., .\rangle_{c, \Delta}$ on $\mathcal{V}_{\Delta}$ uniquely determined by the relations $\left\langle\nu_{\Delta}, \nu_{\Delta}\right\rangle=1$ and $\left(L_{m}\right)^{\dagger}=$ $L_{-m},\left(S_{k}\right)^{\dagger}=S_{-k}$. It is block-diagonal with respect to the $\frac{1}{2} \mathbb{Z}$-grading. We denote by $B_{c, \Delta}^{f}$ the matrix of $\langle., .\rangle_{c, \Delta}$ on $\mathcal{V}_{\Delta}^{f}$ calculated in the basis (3.2):

$$
\left[B_{c, \Delta}^{f}\right]_{K M, L N}=\left\langle\nu_{\Delta, K M}, \nu_{\Delta, L N}\right\rangle_{c, \Delta} \text {. }
$$

It is nonsingular if and only if the supermodule $\mathcal{V}_{\Delta}$ does not contain singular vectors of degrees $\frac{1}{2}, 1, \ldots, f$. The determinant of this matrix is given by the Kac theorem

$$
\operatorname{det} B_{c, \Delta}^{f}=K_{f} \prod_{1 \leqslant r s \leqslant 2 f}\left(\Delta-\Delta_{r s}\right)^{P_{N S}\left(f-\frac{r s}{2}\right)}
$$

where $K_{f}$ depends only on the level $f$, the sum $r+s$ must be even and

$$
\begin{aligned}
\Delta_{r s}(c) & =-\frac{r s-1}{4}+\frac{r^{2}-1}{8} \beta^{2}+\frac{s^{2}-1}{8} \frac{1}{\beta^{2}}, \\
\beta & =\frac{1}{2 \sqrt{2}}(\sqrt{1-\hat{c}}+\sqrt{9-\hat{c}}), \quad \hat{c}=\frac{2}{3} c .
\end{aligned}
$$

The multiplicity of each zero is given by $P_{N S}(f)=\operatorname{dim} \mathcal{V}_{\Delta}^{f}$ and can be read off from the relation

$$
\sum_{f=0}^{\infty} P_{N S}(f) q^{f}=\prod_{n=1}^{\infty} \frac{1+q^{n-\frac{1}{2}}}{1-q^{n}} .
$$

As a function of $c$ the Kac determinant vanishes at

$$
c=c_{r s}(\Delta) \equiv \frac{3}{2}-3\left(\beta_{r s}(\Delta)-\frac{1}{\beta_{r s}(\Delta)}\right)^{2},
$$

where $1<r s \leq 2 n, 1<r, r+s \in 2 \mathbb{N}$, and

$$
\beta_{r s}^{2}(\Delta)=\frac{1}{r^{2}-1}\left(4 \Delta+r s-1+\sqrt{16 \Delta^{2}+8(r s-1) \Delta+(r-s)^{2}}\right) .
$$

We shall use the following parametrization of conformal weights which is especially useful in formulation of the fusion rules:

$$
\Delta_{i}=\frac{\hat{c}-1}{16}+\frac{\alpha_{i}^{2}}{8}=-\frac{1}{8}\left(\beta-\frac{1}{\beta}\right)^{2}+\frac{\alpha_{i}^{2}}{8} .
$$

In the case of the degenerate weight one has:

$$
\Delta_{r s}=\frac{\hat{c}-1}{16}+\frac{\alpha_{r s}^{2}}{8}, \quad \alpha_{r s}=r \beta-\frac{s}{\beta} .
$$




\section{NS chiral vertex operator}

Super-descendants $\varphi_{\Delta, \bar{\Delta}}(\xi, \bar{\xi} \mid z, \bar{z})$ of the super-primary field $\varphi_{\Delta, \bar{\Delta}}(z, \bar{z})=\varphi_{\Delta, \bar{\Delta}}(\nu, \bar{\nu} \mid z, \bar{z})$ are defined by the relations:

$$
\begin{aligned}
\varphi_{\Delta, \bar{\Delta}}\left(L_{-m} \xi, \bar{\xi} \mid z, \bar{z}\right) & =\oint \frac{d w}{2 \pi i}(w-z)^{1-m} T(w) \varphi_{\Delta, \bar{\Delta}}(\xi, \bar{\xi} \mid z, \bar{z}), \quad m \in \mathbb{N}, \\
\varphi_{\Delta, \bar{\Delta}}\left(S_{-k} \xi, \bar{\xi} \mid z, \bar{z}\right) & =\oint \frac{d w}{2 \pi i}(w-z)^{\frac{1}{2}-k} S(w) \varphi_{\Delta, \bar{\Delta}}(\xi, \bar{\xi} \mid z, \bar{z}), \quad k \in \mathbb{N}-\frac{1}{2},
\end{aligned}
$$

and by analogous formulae for the right sector. Using conformal Ward identities one can express an arbitrary correlator of three descendants in terms of matrix elements which can be further factorized into the holomorphic and anti-holomorphic parts

$$
\left\langle\xi_{3}, \bar{\xi}_{3}\left|\varphi_{\Delta_{2}, \bar{\Delta}_{2}}\left(\xi_{2}, \bar{\xi}_{2} \mid z, \bar{z}\right)\right| \xi_{1}, \bar{\xi}_{1}\right\rangle=\varrho_{\infty}^{\Delta_{3} \Delta_{2} \Delta_{1}}\left(\xi_{1}, \xi_{2}, \xi_{3}\right) \varrho_{\infty}^{\bar{\Delta}_{3} \bar{\Delta}_{2} \bar{\Delta}_{1}} \overline{\bar{z}}_{0}\left(\bar{\xi}_{1}, \bar{\xi}_{2}, \bar{\xi}_{3}\right),
$$

where $\xi_{i} \in \mathcal{V}_{\Delta_{i}}, \bar{\xi}_{i} \in \mathcal{V}_{\bar{\Delta}_{i}}$. The trilinear map

$$
\varrho_{\infty}^{\Delta_{3} \Delta_{2} \Delta_{1}{ }_{0}}: \mathcal{V}_{\Delta_{3}} \times \mathcal{V}_{\Delta_{2}} \times \mathcal{V}_{\Delta_{1}} \mapsto \mathbb{C}
$$

is determined by conditions which can be easily derived by analyzing the holomorphic (or anti-holomorphic) part of the superconformal Ward identities for the three point function (see [8] for an analogous construction in the Virasoro case). They read:

$$
\begin{aligned}
\varrho_{\infty}^{\Delta_{3} \Delta_{2} \Delta_{1}}\left(L_{-n} \xi_{3}, \xi_{2}, \xi_{1}\right) & =\varrho_{\infty}^{\Delta_{3} \Delta_{2} \Delta_{1}}\left(\xi_{3}, \xi_{2}, L_{n} \xi_{1}\right) \\
& +\sum_{m=-1}^{l(n)}\left(\begin{array}{c}
n+1 \\
m+1
\end{array}\right) z^{n-m} \varrho_{\infty}^{\Delta_{3} \Delta_{2} \Delta_{1}}\left(\xi_{3}, L_{m} \xi_{2}, \xi_{1}\right) \\
\varrho_{\infty}^{\Delta_{3} \Delta_{2} \Delta_{1}}\left(S_{-k} \xi_{3}, \xi_{2}, \xi_{1}\right) & =(-1)^{2\left(N\left(\xi_{1}\right)+N\left(\xi_{3}\right)\right)} \varrho_{\infty}^{\Delta_{3} \Delta_{2} \Delta_{1}}\left(\xi_{3}, \xi_{2}, S_{k} \xi_{1}\right) \\
& +\sum_{m=-1}^{l\left(k-\frac{1}{2}\right)}\left(\begin{array}{c}
k+\frac{1}{2} \\
m+1
\end{array}\right) z^{k-\frac{1}{2}-m} \varrho_{\infty}^{\Delta_{3} \Delta_{2} \Delta_{1}}\left(\xi_{3}, S_{m+\frac{1}{2}} \xi_{2}, \xi_{1}\right)
\end{aligned}
$$

where $l(m)=m$ for $m+1 \geqslant 0$, and $l(m)=\infty$ for $m+1<0$, and

$$
\begin{aligned}
& \varrho_{\infty}^{\Delta_{3} \Delta_{2} \Delta_{1} \Delta_{1}}\left(\xi_{3}, L_{-1} \xi_{2}, \xi_{1}\right)=\partial_{z} \varrho_{\infty}^{\Delta_{3} \Delta_{2} \Delta_{1} \Delta_{1}}\left(\xi_{3}, \xi_{2}, \xi_{1}\right) \\
& \varrho_{\infty}^{\Delta_{3} \Delta_{2} \Delta_{1}}\left(\xi_{3}, L_{n} \xi_{2}, \xi_{1}\right)=\sum_{m=0}^{n+1}\left(\begin{array}{c}
n+1 \\
m
\end{array}\right)(-z)^{m}\left(\varrho_{\infty}^{\Delta_{3} \Delta_{2} \Delta_{1}}\left(L_{m-n} \xi_{3}, \xi_{2}, \xi_{1}\right)\right. \\
& \left.-\varrho_{\infty}^{\Delta_{3} \Delta_{2} \Delta_{1} \Delta_{1}}\left(\xi_{3}, \xi_{2}, L_{n-m} \xi_{1}\right)\right), \quad n>-1 \\
& \varrho_{\infty}^{\Delta_{3} \Delta_{2} \Delta_{1}}\left(\xi_{3}, L_{-n} \xi_{2}, \xi_{1}\right)=\sum_{m=0}^{\infty}\left(\begin{array}{c}
n-2+m \\
n-2
\end{array}\right) z^{m} \varrho_{\infty}^{\Delta_{3} \Delta_{2} \Delta_{1}}\left(L_{n+m} \xi_{3}, \xi_{2}, \xi_{1}\right) \\
& +(-1)^{n} \sum_{m=0}^{\infty}\left(\begin{array}{c}
n-2+m \\
n-2
\end{array}\right) z^{-n+1-m} \varrho_{\infty}^{\Delta_{3} \Delta_{2} \Delta_{1}}\left(\xi_{3}, \xi_{2}, L_{m-1} \xi_{1}\right), \quad n>1
\end{aligned}
$$




$$
\begin{aligned}
& \varrho_{\infty}^{\Delta_{3} \Delta_{2} \Delta_{1}}\left(\xi_{3}, S_{k} \xi_{2}, \xi_{1}\right)=\sum_{m=0}^{k+\frac{1}{2}}\left(\begin{array}{c}
k+\frac{1}{2} \\
m
\end{array}\right)(-z)^{m}\left(\varrho_{\infty}^{\Delta_{3} \Delta_{2} \Delta_{1}}\left(S_{m-k} \xi_{3}, \xi_{2}, \xi_{1}\right)\right. \\
& \left.+(-1)^{2\left(N\left(\xi_{1}\right)+N\left(\xi_{3}\right)\right)} \varrho_{\infty}^{\Delta_{3} \Delta_{2} \Delta_{1}}\left(\xi_{3}, \xi_{2}, S_{k-m} \xi_{1}\right)\right), \quad k \geqslant-\frac{1}{2}, \\
& \varrho_{\infty}^{\Delta_{3} \Delta_{2} \Delta_{1}}\left(\xi_{3}, S_{-k} \xi_{2}, \xi_{1}\right)=\sum_{m=0}^{\infty}\left(\begin{array}{c}
k-\frac{3}{2}+m \\
m
\end{array}\right) z^{m} \varrho_{\infty}^{\Delta_{3} \Delta_{2} \Delta_{1}}\left(S_{k+m} \xi_{3}, \xi_{2}, \xi_{1}\right) \\
& +(-1)^{2\left(N\left(\xi_{1}\right)+N\left(\xi_{3}\right)\right)+k+\frac{1}{2}} \sum_{m=0}^{\infty}\left(\begin{array}{c}
k-\frac{3}{2}+m \\
m
\end{array}\right) z^{-k-m+\frac{1}{2}} \varrho_{\infty}^{\Delta_{3} \Delta_{2} \Delta_{1}}{ }_{0}\left(\xi_{3}, \xi_{2}, S_{m-\frac{1}{2}} \xi_{1}\right), \quad k>\frac{1}{2} .
\end{aligned}
$$

The form $\varrho_{\infty}^{\Delta_{3} \Delta_{2} \Delta_{1}}$ is almost completely determined by the properties above. In particular, for $L_{0}$-eingenstates, $L_{0} \xi_{i}=\Delta_{i}\left(\xi_{i}\right) \xi_{i}, i=1,2,3$, one has:

$$
\varrho_{\infty}^{\Delta_{3} \Delta_{2} \Delta_{1} \Delta_{1}}\left(\xi_{3}, \xi_{2}, \xi_{1}\right)=z^{\Delta_{3}\left(\xi_{3}\right)-\Delta_{2}\left(\xi_{2}\right)-\Delta_{1}\left(\xi_{1}\right)} \varrho_{\infty}^{\Delta_{3} \Delta_{2} \Delta_{1}}\left(\xi_{3}, \xi_{2}, \xi_{1}\right)
$$

However, in contrast to the case of the Virasoro algebra, the form $\varrho_{\infty}^{\Delta_{3}} \Delta_{1}^{\Delta_{2}} \begin{gathered}\Delta_{1} \\ 0\end{gathered}$ is determined up to two, instead of one, independent constants:

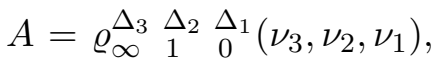

$$
\begin{aligned}
& B=\varrho_{\infty}^{\Delta_{3}} \underset{1}{\Delta_{2}} \underset{0}{\Delta_{1}}\left(\nu_{3}, * \nu_{2}, \nu_{1}\right)=\varrho_{\infty}^{\Delta_{3}} \underset{1}{\Delta_{2}} \underset{0}{\Delta_{1}}\left(* \nu_{3}, \nu_{2}, \nu_{1}\right)=\varrho_{\infty}^{\Delta_{3}} \underset{1}{\Delta_{2}} \underset{0}{\Delta_{1}}\left(\nu_{3}, \nu_{2}, * \nu_{1}\right),
\end{aligned}
$$

where $\nu_{i}$ is the highest weight state in $\mathcal{V}_{\Delta_{i}}(i=1,2,3)$ and

$$
* \nu_{i} \equiv S_{-\frac{1}{2}} \nu_{i}
$$

For this reason the NS superconformal theory requires two independent structure constants (and in consequence 8 conformal blocks). Indeed, taking into account that the superprimary fields are even with respect to the common parity operator $(-1)^{F} \otimes(-1)^{\bar{F}}$ one can show that the constants $A_{L}, B_{L}$ of the left sector and the constants $A_{R}, B_{R}$ of the right sector always show up in the combinations $A_{L} A_{R}, B_{L} B_{R}$ which are just the structure constants (2.7), (2.8):

$$
\begin{aligned}
& A_{L} A_{R}=C_{321}=\left\langle\varphi_{3}(\infty, \infty) \varphi_{2}(1,1) \varphi_{1}(0,0)\right\rangle, \\
& B_{L} B_{R}=\widetilde{C}_{321}=\left\langle\varphi_{3}(\infty, \infty) \widetilde{\varphi}_{2}(1,1) \varphi_{1}(0,0)\right\rangle .
\end{aligned}
$$

As it is more convenient to keep in the correlation functions an explicit $C_{321}$ and $\widetilde{C}_{321}$ dependence we shall work in the following mostly with the normalized form. In order to avoid confusion with the unnormalized form $\varrho_{\infty}^{\Delta_{3}} \underset{z}{\Delta_{2}} \underset{0}{\Delta_{1}}$ we shall denote the normalized one by $\rho_{\infty}^{\Delta_{3}} \underset{z}{\Delta_{2}} \stackrel{\Delta_{1}}{\Delta_{1}}$. Thus, by definition

$$
\rho_{\infty}^{\Delta_{3}} \underset{z}{\Delta_{2}} \underset{0}{\Delta} \Delta_{1}\left(\nu_{3}, \nu_{2}, \nu_{1}\right)=\rho_{\infty}^{\Delta_{3}} \underset{z}{\Delta_{2}} \underset{0}{\Delta} \Delta_{1}\left(\nu_{3}, * \nu_{2}, \nu_{1}\right)=1
$$


For each $\xi_{2} \in \mathcal{V}_{\Delta_{2}}$ we define the (generalized) chiral vertex $V\left(\xi_{2} \mid z\right)$ [9] as a linear map

$$
V\left(\xi_{2} \mid z\right): \mathcal{V}_{\Delta_{1}} \mapsto \mathcal{V}_{\Delta_{3}}
$$

such that

$$
\left\langle\xi_{3}\left|V\left(\xi_{2} \mid z\right)\right| \xi_{1}\right\rangle=\rho_{\infty}^{\Delta_{3} \Delta_{2} \Delta_{1}}\left(\xi_{3}, \xi_{2}, \xi_{1}\right)
$$

for all $\xi_{3} \in \mathcal{V}_{\Delta_{3}}, \xi_{1} \in \mathcal{V}_{\Delta_{1}}$. Note that $V\left(\xi_{2} \mid z\right)$ does not have definite parity with respect to the left fermionic number $(-1)^{F}$ and can be decomposed into its even (parity preserving) and odd (parity reversing) part:

$$
V\left(\xi_{2} \mid z\right)=V^{\text {even }}\left(\xi_{2} \mid z\right)+V^{\text {odd }}\left(\xi_{2} \mid z\right) .
$$

We shall need two special cases of these operators: $V\left(\nu_{2} \mid z\right)$ and $V\left(* \nu_{2} \mid z\right)$. Formulae (4.2), (4.3) and (4.4) imply the following relations:

$$
\begin{aligned}
{\left[\mathrm{L}_{m}, V\left(\nu_{2} \mid z\right)\right] } & =z^{m}\left(z \partial_{z}+(m+1) \Delta_{2}\right) V\left(\nu_{2} \mid z\right), \\
{\left[\mathrm{L}_{m}, V\left(* \nu_{2} \mid z\right)\right] } & =z^{m}\left(z \partial_{z}+(m+1)\left(\Delta_{2}+\frac{1}{2}\right)\right) V\left(* \nu_{2} \mid z\right), \\
{\left[\mathrm{S}_{k}, V^{\text {even }}\left(\nu_{2} \mid z\right)\right] } & =z^{k+\frac{1}{2}} V^{\text {odd }}\left(* \nu_{2} \mid z\right), \\
\left\{\mathrm{S}_{k}, V^{\text {odd }}\left(\nu_{2} \mid z\right)\right\} & =z^{k+\frac{1}{2}} V^{\text {even }}\left(* \nu_{2} \mid z\right), \\
{\left[\mathrm{S}_{k}, V^{\text {even }}\left(* \nu_{2} \mid z\right)\right] } & =z^{k-\frac{1}{2}}\left(z \partial_{z}+\Delta_{2}(2 k+1)\right) V^{\text {odd }}\left(\nu_{2} \mid z\right), \\
\left\{\mathrm{S}_{k}, V^{\text {odd }}\left(* \nu_{2} \mid z\right)\right\} & =z^{k-\frac{1}{2}}\left(z \partial_{z}+\Delta_{2}(2 k+1)\right) V^{\text {even }}\left(\nu_{2} \mid z\right) .
\end{aligned}
$$

Using them one gets:

$$
\begin{aligned}
& \rho_{\infty}^{\Delta_{3}} \underset{z}{\Delta_{2}} \underset{0}{\Delta_{1}}\left(\nu_{3, K M}, \nu_{2}, \nu_{1}\right)=z^{\Delta_{3}+|K|+|M|-\Delta_{2}-\Delta_{1}}\left\{\begin{array}{l}
\eta_{\Delta_{3}+|M|}^{\mathrm{o}}\left[\begin{array}{c}
\Delta_{2} \\
\Delta_{1}
\end{array}\right]_{K} \gamma_{\Delta_{3}}\left[\begin{array}{c}
\Delta_{2} \\
\Delta_{1}
\end{array}\right]_{M}, \\
\eta_{\Delta_{3}+|M|}^{\mathrm{e}}\left[\begin{array}{c}
\Delta_{2} \\
\Delta_{1}
\end{array}\right]_{K} \gamma_{\Delta_{3}}\left[\begin{array}{c}
\Delta_{2}+\frac{1}{2} \\
\Delta_{1}
\end{array}\right]_{M},
\end{array}\right. \\
& \rho_{\infty}^{\Delta_{3}} \underset{z}{\Delta_{2}} \underset{\Delta_{1}}{\Delta_{1}}\left(\nu_{3, K M}, * \nu_{2}, \nu_{1}\right)=z^{\Delta_{3}+|K|+|M|-\Delta_{2}-\Delta_{1}-\frac{1}{2}}\left\{\begin{array}{l}
\eta_{\Delta_{3}+|M|}^{\mathrm{e}}\left[\begin{array}{c}
\Delta_{2} \\
\Delta_{1}
\end{array}\right]_{K} \gamma_{\Delta_{3}}\left[\begin{array}{c}
\Delta_{2}+\frac{1}{2} \\
\Delta_{1}
\end{array}\right]_{M}, \\
\eta_{\Delta_{3}+|M|}^{\mathrm{o}}\left[\begin{array}{c}
\Delta_{2} \\
\Delta_{1}
\end{array}\right]_{K} \gamma_{\Delta_{3}}\left[\begin{array}{c}
\Delta_{2} \\
\Delta_{1}
\end{array}\right]_{M},
\end{array}\right.
\end{aligned}
$$

where the upper lines correspond to $|K| \in \mathbb{N}$, the lower lines to $|K| \in \mathbb{N}-\frac{1}{2}$, and

$$
\begin{aligned}
& \gamma_{\Delta}\left[\begin{array}{l}
\Delta_{2} \\
\Delta_{1}
\end{array}\right]_{M} \stackrel{\text { def }}{=}\left(\Delta-\Delta_{1}+m_{1} \Delta_{2}\right)\left(\Delta-\Delta_{1}+m_{2} \Delta_{2}+m_{1}\right) \cdots\left(\Delta-\Delta_{1}+m_{j} \Delta_{2}+\sum_{l=1}^{j-1} m_{l}\right), \\
& \eta_{\Delta}^{\mathrm{o}}\left[\begin{array}{c}
\Delta_{2} \\
\Delta_{1}
\end{array}\right]_{K} \stackrel{\text { def }}{=}\left(\Delta-\Delta_{1}+2 k_{1} \Delta_{2}\right)\left(\Delta-\Delta_{1}+2 k_{3} \Delta_{2}+k_{1}+k_{2}\right) \ldots\left(\Delta-\Delta_{1}+2 k_{p} \Delta_{2}+\sum_{l=1}^{p-1} k_{l}\right), \\
& \eta_{\Delta}^{\mathrm{e}}\left[\begin{array}{c}
\Delta_{2} \\
\Delta_{1}
\end{array}\right]_{K} \stackrel{\text { def }}{=}\left(\Delta-\Delta_{1}+2 k_{2} \Delta_{2}+k_{1}\right)\left(\Delta-\Delta_{1}+2 k_{4} \Delta_{2}+\sum_{l=1}^{3} k_{l}\right) . .\left(\Delta-\Delta_{1}+2 k_{p^{\prime}} \Delta_{2}+\sum_{l=1}^{p^{\prime}-1} k_{l}\right),
\end{aligned}
$$


where $p$ is the largest odd number not grater than $i$, and $p^{\prime}$ is the largest even number not grater than $i$.

For the matrix elements $\left\langle\nu_{3}\left|V\left(\_\nu_{2} \mid z\right)\right| \nu_{1, K M}\right\rangle$ one gets:

$$
\begin{aligned}
& \rho_{\infty}^{\Delta_{3}} \underset{1}{\Delta_{2}} \underset{0}{\Delta_{1}}\left(\nu_{3}, \nu_{2}, \nu_{1, K M}\right)=\rho_{\infty}^{\Delta_{1}} \stackrel{1}{1}_{2} \underset{0}{\Delta_{3}}\left(\nu_{1, K M}, \nu_{3}, \nu_{4}\right), \\
& \rho_{\infty}^{\Delta_{3}} \begin{array}{ccc}
\Delta_{2} & \Delta_{1} & 0
\end{array}\left(\nu_{3}, * \nu_{2}, \nu_{1, K M}\right)=\rho_{\infty}^{\Delta_{1}} \underset{1}{\Delta_{2}} \underset{0}{\Delta_{3}}\left(\nu_{1, K M}, * \nu_{2}, \nu_{3}\right),
\end{aligned}
$$

for $|K| \in \mathbb{N} \cup\{0\}$, and

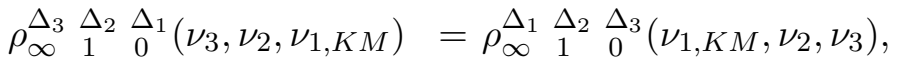

$$
\begin{aligned}
& \rho_{\infty}^{\Delta_{3}} \underset{1}{\Delta_{2}} \underset{0}{\Delta_{1}}\left(\nu_{3}, * \nu_{2}, \nu_{1, K M}\right)=-\rho_{\infty}^{\Delta_{1}} \underset{1}{\Delta_{2}} \underset{0}{\Delta} \Delta_{3}\left(\nu_{1, K M}, * \nu_{2}, \nu_{3}\right),
\end{aligned}
$$

for $|K| \in \mathbb{N}-\frac{1}{2}$.

Using formulae (4.12) and (4.13) one can show an important factorization property of the form $\varrho_{\infty}^{\Delta_{3}} \Delta_{2} \Delta_{2} \Delta_{1}$. For an arbitrary $L_{0}$-eingenstate $L_{0} \xi_{3}=\left(\Delta_{3}+f\right) \xi_{3}$ one has:

$$
\begin{aligned}
& \varrho_{\infty}^{\Delta_{3}} \underset{z}{\Delta_{2}} \underset{0}{\Delta_{1}}\left(\mathrm{~S}_{-K} \mathrm{~L}_{-M} \xi_{3}, \nu_{2}, \nu_{1}\right)=z^{\Delta_{3}+f+|K|+|M|-\Delta_{2}-\Delta_{1}} \times
\end{aligned}
$$

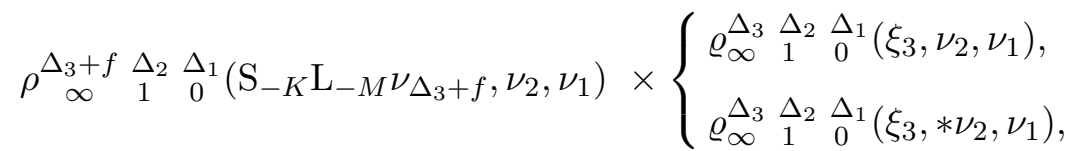

$$
\begin{aligned}
& \varrho_{\infty}^{\Delta_{3}} \underset{z}{\Delta_{2}}{ }_{0}^{\Delta_{1}}\left(\mathrm{~S}_{-K} \mathrm{~L}_{-M} \xi_{3}, * \nu_{2}, \nu_{1}\right)=z^{\Delta_{3}+f+|K|+|M|-\Delta_{2}-\Delta_{1}-\frac{1}{2}} \times
\end{aligned}
$$

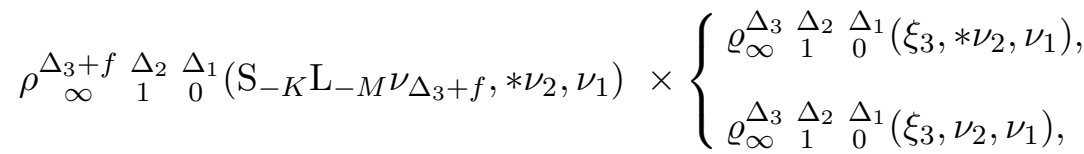

where the upper lines correspond to $|K| \in \mathbb{N} \cup\{0\}$, and the lower lines to $|K| \in \mathbb{N}-\frac{1}{2}$.

\section{NS superconformal blocks}

W shall define four types of NS superconformal blocks directly in terms of the form $\rho$. For each type there is one even,

$$
\mathcal{F}_{\Delta}^{1}\left[\begin{array}{cc}
-\Delta_{3} & \Delta_{2} \\
\Delta_{4} & \Delta_{1}
\end{array}\right](z)=z^{\Delta-{ }_{-} \Delta_{2}-\Delta_{1}}\left(1+\sum_{m \in \mathbb{N}} z^{m} F_{c, \Delta}^{m}\left[\begin{array}{cc}
-\Delta_{3}-\Delta_{2} \\
\Delta_{4} & \Delta_{1}
\end{array}\right]\right),
$$

and one odd,

$$
\mathcal{F}_{\Delta}^{\frac{1}{2}}\left[\begin{array}{cc}
-\Delta_{3} & \Delta_{2} \\
\Delta_{4} & \Delta_{1}
\end{array}\right](z)=z^{\Delta-{ }_{-} \Delta_{2}-\Delta_{1}} \sum_{k \in \mathbb{N}-\frac{1}{2}} z^{k} F_{c, \Delta}^{k}\left[\begin{array}{cc}
-\Delta_{3}-\Delta_{2} \\
\Delta_{4} & \Delta_{1}
\end{array}\right]
$$

conformal block. 
The coefficients are defined by:

$$
\begin{aligned}
& F_{c, \Delta}^{f}\left[\begin{array}{cc}
-\Delta_{3} & \Delta_{2} \\
\Delta_{4} & \Delta_{1}
\end{array}\right]= \\
& =\sum \rho_{\infty}^{\Delta_{4}} \underset{3}{\Delta_{3}} \underset{0}{\Delta}\left(\nu_{4}, \nu_{-} \nu_{3}, \nu_{\Delta, K M}\right)\left[B_{c, \Delta}^{f}\right]^{K M, L N} \rho_{\infty}^{\Delta} \underset{1}{\Delta_{2}} \underset{0}{\Delta_{1}}\left(\nu_{\Delta, L N},{ }_{-} \nu_{2}, \nu_{1}\right), \\
& |K|+|M|=|L|+|N|=f
\end{aligned}
$$

where $\left[B_{c, \Delta}^{f}\right]^{K M, L N}$ is the matrix inverse to the matrix $(3.3),{ }_{-} \Delta_{i}$ and $\_\nu_{i}$ stand for $\Delta_{i}$ or $* \Delta_{i}$, and $\nu_{i}$ or $* \nu_{i}$, respectively, and $z^{\Delta-* \Delta_{2}-\Delta_{1}}=z^{\Delta-\Delta_{2}-\Delta_{1}-\frac{1}{2}}$. For instance:

$$
\begin{aligned}
& \mathcal{F}_{\Delta}^{1}\left[\begin{array}{rr}
\Delta_{3} & * \Delta_{2} \\
\Delta_{4} & \Delta_{1}
\end{array}\right](z)=z^{\Delta-\Delta_{2}-\Delta_{1}-\frac{1}{2}}\left(1+\sum_{m \in \mathbb{N}} z^{m} F_{c, \Delta}^{m}\left[\begin{array}{cc}
\Delta_{3} * \Delta_{2} \\
\Delta_{4} & \Delta_{1}
\end{array}\right]\right),
\end{aligned}
$$

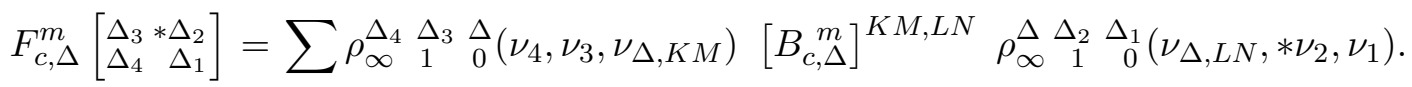

Let us note that the definition above is independent of the choice of basis in $\mathcal{V}_{\Delta}^{f}$.

The formulae (4.14), (4.15) imply simple relations:

$$
\begin{aligned}
& \mathcal{F}_{\Delta}^{1}\left[\begin{array}{cc}
\Delta_{3} & * \Delta_{2} \\
\Delta_{4} & \Delta_{1}
\end{array}\right](z)=z^{-\Delta_{2}-\Delta_{1}-\frac{1}{2}+\Delta_{4}+\Delta_{3}} \quad \mathcal{F}_{\Delta}^{1}\left[\begin{array}{cc}
* \Delta_{2} & \Delta_{3} \\
\Delta_{1} & \Delta_{4}
\end{array}\right](z), \\
& \mathcal{F}_{\Delta}^{\frac{1}{2}}\left[\begin{array}{cc}
\Delta_{3} & * \Delta_{2} \\
\Delta_{4} & \Delta_{1}
\end{array}\right](z)=-z^{-\Delta_{2}-\Delta_{1}-\frac{1}{2}+\Delta_{4}+\Delta_{3}} \mathcal{F}_{\Delta}^{\frac{1}{2}}\left[\begin{array}{rr}
* \Delta_{2} & \Delta_{3} \\
\Delta_{1} & \Delta_{4}
\end{array}\right](z),
\end{aligned}
$$

reducing the number of blocks to 6 independent functions.

The primary fields are expressed in terms of chiral vertex operators as follows:

$$
\begin{aligned}
& \phi_{2}(z, \bar{z})=\bigoplus_{\Delta_{3}, \Delta_{1}}\left(C_{321} V^{\text {even }}\left(\nu_{2} \mid z\right) \otimes V^{\text {even }}\left(\bar{\nu}_{2} \mid \bar{z}\right)-\tilde{C}_{321} V^{\text {odd }}\left(\nu_{2} \mid z\right) \otimes V^{\text {odd }}\left(\bar{\nu}_{2} \mid \bar{z}\right)\right), \\
& \psi_{2}(z, \bar{z})=\bigoplus_{\Delta_{3}, \Delta_{1}}\left(C_{321} V^{\text {odd }}\left(* \nu_{2} \mid z\right) \otimes V^{\text {even }}\left(\bar{\nu}_{2} \mid \bar{z}\right)-\tilde{C}_{321} V^{\text {even }}\left(* \nu_{2} \mid z\right) \otimes V^{\text {odd }}\left(\bar{\nu}_{2} \mid \bar{z}\right)\right), \\
& \bar{\psi}_{2}(z, \bar{z})=\bigoplus_{\Delta_{3}, \Delta_{1}}\left(C_{321} V^{\text {even }}\left(\nu_{2} \mid z\right) \otimes V^{\text {odd }}\left(* \bar{\nu}_{2} \mid \bar{z}\right)+\tilde{C}_{321} V^{\text {odd }}\left(\nu_{2} \mid z\right) \otimes V^{\text {even }}\left(* \bar{\nu}_{2} \mid \bar{z}\right)\right), \\
& \tilde{\phi}_{2}(z, \bar{z})=\bigoplus_{\Delta_{3}, \Delta_{1}}\left(C_{321} V^{\text {odd }}\left(* \nu_{2} \mid z\right) \otimes V^{\text {odd }}\left(* \bar{\nu}_{2} \mid \bar{z}\right)+\tilde{C}_{321} V^{\text {even }}\left(* \nu_{2} \mid z\right) \otimes V^{\text {even }}\left(* \bar{\nu}_{2} \mid \bar{z}\right)\right) .
\end{aligned}
$$

Using this representation and factorization in the (not orthogonal) basis (3.2) one gets the following expressions for all basic 4-point functions (for simplicity we write the expressions in the diagonal case $\Delta_{i}=\bar{\Delta}_{i}$ ): 
$\left\langle\Delta_{4}\left|\phi_{3}(1,1) \phi_{2}(z, \bar{z})\right| \Delta_{1}\right\rangle=$

$$
\sum_{p}\left(C_{43 p} C_{p 21}\left|\mathcal{F}_{\Delta_{p}}^{1}\left[\begin{array}{ll}
\Delta_{3} & \Delta_{2} \\
\Delta_{4} & \Delta_{1}
\end{array}\right](z)\right|^{2}-\tilde{C}_{43 p} \tilde{C}_{p 21}\left|\mathcal{F}_{\Delta_{p}}^{\frac{1}{2}}\left[\begin{array}{cc}
\Delta_{3} & \Delta_{2} \\
\Delta_{4} & \Delta_{1}
\end{array}\right](z)\right|^{2}\right),
$$

$\left\langle\Delta_{4}\left|\phi_{3}(1,1) \tilde{\phi}_{2}(z, \bar{z})\right| \Delta_{1}\right\rangle=$

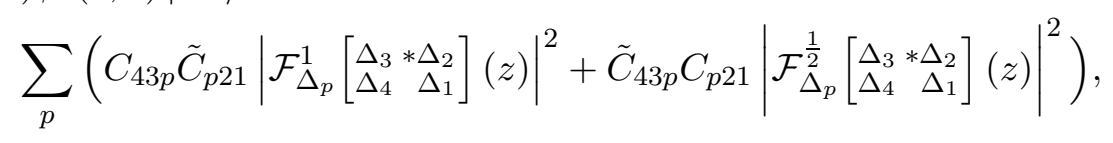

$\left\langle\Delta_{4}\left|\tilde{\phi}_{3}(1,1) \phi_{2}(z, \bar{z})\right| \Delta_{1}\right\rangle=$

$$
\sum_{p}\left(\tilde{C}_{43 p} C_{p 21}\left|\mathcal{F}_{\Delta_{p}}^{1}\left[\begin{array}{rr}
* \Delta_{3} & \Delta_{2} \\
\Delta_{4} & \Delta_{1}
\end{array}\right](z)\right|^{2}+C_{43 p} \tilde{C}_{p 21}\left|\mathcal{F}_{\Delta_{p}}^{\frac{1}{2}}\left[\begin{array}{rr}
* \Delta_{3} & \Delta_{2} \\
\Delta_{4} & \Delta_{1}
\end{array}\right](z)\right|^{2}\right)
$$

$\left\langle\Delta_{4}\left|\tilde{\phi}_{3}(1,1) \tilde{\phi}_{2}(z, \bar{z})\right| \Delta_{1}\right\rangle=$

$$
\sum_{p}\left(\tilde{C}_{43 p} \tilde{C}_{p 21}\left|\mathcal{F}_{\Delta_{p}}^{1}\left[\begin{array}{rr}
* \Delta_{3} * \Delta_{2} \\
\Delta_{4} & \Delta_{1}
\end{array}\right](z)\right|^{2}-C_{43 p} C_{p 21}\left|\mathcal{F}_{\Delta_{p}}^{\frac{1}{2}}\left[\begin{array}{rr}
* \Delta_{3} * \Delta_{2} \\
\Delta_{4} & \Delta_{1}
\end{array}\right](z)\right|^{2}\right)
$$

$\left\langle\Delta_{4}\left|\psi_{3}(1,1) \psi_{2}(z, \bar{z})\right| \Delta_{1}\right\rangle=$

$$
\begin{aligned}
& \sum_{p}\left(\tilde{C}_{43 p} \tilde{C}_{p 21} \mathcal{F}_{\Delta_{p}}^{1}\left[\begin{array}{cc}
* \Delta_{3} & * \Delta_{2} \\
\Delta_{4} & \Delta_{1}
\end{array}\right](z) \mathcal{F}_{\Delta_{p}}^{\frac{1}{2}}\left[\begin{array}{ll}
\Delta_{3} & \Delta_{2} \\
\Delta_{4} & \Delta_{1}
\end{array}\right](\bar{z})\right. \\
& \left.+C_{43 p} C_{p 21} \mathcal{F}_{\Delta_{p}}^{\frac{1}{2}}\left[\begin{array}{cc}
* \Delta_{3} * \Delta_{2} \\
\Delta_{4} & \Delta_{1}
\end{array}\right](z) \mathcal{F}_{\Delta_{p}}^{1}\left[\begin{array}{ll}
\Delta_{3} & \Delta_{2} \\
\Delta_{4} & \Delta_{1}
\end{array}\right](\bar{z})\right)
\end{aligned}
$$

$\left\langle\Delta_{4}\left|\bar{\psi}_{3}(1,1) \bar{\psi}_{2}(z, \bar{z})\right| \Delta_{1}\right\rangle=$

$$
\begin{aligned}
& \sum_{p}\left(C_{43 p} C_{p 21} \mathcal{F}_{\Delta_{p}}^{1}\left[\begin{array}{ll}
\Delta_{3} & \Delta_{2} \\
\Delta_{4} & \Delta_{1}
\end{array}\right](z) \mathcal{F}_{\Delta_{p}}^{\frac{1}{2}}\left[\begin{array}{cc}
* \Delta_{3} * \Delta_{2} \\
\Delta_{4} & \Delta_{1}
\end{array}\right](\bar{z})\right. \\
& \left.+\tilde{C}_{43 p} \tilde{C}_{p 21} \mathcal{F}_{\Delta_{p}}^{\frac{1}{2}}\left[\begin{array}{ll}
\Delta_{3} & \Delta_{2} \\
\Delta_{4} & \Delta_{1}
\end{array}\right](z) \mathcal{F}_{\Delta_{p}}^{1}\left[\begin{array}{rr}
* \Delta_{3} * \Delta_{2} \\
\Delta_{4} & \Delta_{1}
\end{array}\right](\bar{z})\right)
\end{aligned}
$$

$\left\langle\Delta_{4}\left|\psi_{3}(1,1) \bar{\psi}_{2}(z, \bar{z})\right| \Delta_{1}\right\rangle=$

$$
\begin{aligned}
\sum_{p}\left(-\tilde{C}_{43 p} C_{p 21} \mathcal{F}_{\Delta_{p}}^{1}\left[\begin{array}{rr}
* \Delta_{3} & \Delta_{2} \\
\Delta_{4} & \Delta_{1}
\end{array}\right](z) \mathcal{F}_{\Delta_{p}}^{\frac{1}{2}}\left[\begin{array}{rr}
\Delta_{3} & * \Delta_{2} \\
\Delta_{4} & \Delta_{1}
\end{array}\right](\bar{z})\right. \\
\left.+C_{43 p} \tilde{C}_{p 21} \mathcal{F}_{\Delta_{p}}^{\frac{1}{2}}\left[\begin{array}{rr}
* \Delta_{3} & \Delta_{2} \\
\Delta_{4} & \Delta_{1}
\end{array}\right](z) \mathcal{F}_{\Delta_{p}}^{1}\left[\begin{array}{rr}
\Delta_{3} & * \Delta_{2} \\
\Delta_{4} & \Delta_{1}
\end{array}\right](\bar{z})\right)
\end{aligned}
$$

$\left\langle\Delta_{4}\left|\bar{\psi}_{3}(1,1) \psi_{2}(z, \bar{z})\right| \Delta_{1}\right\rangle=$

$$
\begin{aligned}
\sum_{p}\left(-C_{43 p} \tilde{C}_{p 21} \mathcal{F}_{\Delta_{p}}^{1}\left[\begin{array}{ll}
\Delta_{3} * \Delta_{2} \\
\Delta_{4} & \Delta_{1}
\end{array}\right](z) \mathcal{F}_{\Delta_{p}}^{\frac{1}{2}}\left[\begin{array}{rr}
* \Delta_{3} & \Delta_{2} \\
\Delta_{4} & \Delta_{1}
\end{array}\right](\bar{z})\right. \\
\left.+\tilde{C}_{43 p} C_{p 21} \mathcal{F}_{\Delta_{p}}^{\frac{1}{2}}\left[\begin{array}{rr}
\Delta_{3} * \Delta_{2} \\
\Delta_{4}
\end{array}\right](z) \mathcal{F}_{\Delta_{p}}^{1}\left[\begin{array}{r}
* \Delta_{3} \Delta_{2} \\
\Delta_{4} \Delta_{1}
\end{array}\right](\bar{z})\right)
\end{aligned}
$$




\section{Recurrence relations}

It follows from the definition of the blocks' coefficients $F_{c, \Delta}^{f}\left[\begin{array}{cc}-\Delta_{3} \Delta_{2} \\ \Delta_{4} \Delta_{1}\end{array}\right]$ that they are polynomials in the external weights $\Delta_{i}$, and rational functions of the intermediate weight $\Delta$ and the central charge $c$. Consequently, they can be expressed either as a sum over the poles in $\Delta$ :

$$
F_{c, \Delta}^{f}\left[\begin{array}{cc}
-\Delta_{3} & \Delta_{2} \\
\Delta_{4} & \Delta_{1}
\end{array}\right]=\mathrm{h}_{c, \Delta}^{f}\left[\begin{array}{cc}
-\Delta_{3} & \Delta_{2} \\
\Delta_{4} & \Delta_{1}
\end{array}\right]+\sum_{\substack{1<r s \leq 2 f \\
r+s \in 2 \mathbb{N}}} \frac{\mathcal{R}_{c, r s}^{f}\left[\begin{array}{c}
-\Delta_{3}-\Delta_{2} \\
\Delta_{4}
\end{array} \Delta_{1}\right.}{\Delta-\Delta_{r s}(c)}
$$

with $\Delta_{r s}(c)$ given by $(3.5)$, or as a sum over the poles in $c$ :

$$
F_{c, \Delta}^{f}\left[\begin{array}{cc}
-\Delta_{3} & \Delta_{2} \\
\Delta_{4} & \Delta_{1}
\end{array}\right]=\mathrm{f}_{\Delta}^{f}\left[\begin{array}{cc}
-\Delta_{3} & \Delta_{2} \\
\Delta_{4} & \Delta_{1}
\end{array}\right]+\sum_{\substack{1<r s \leq 2 f, r>1 \\
r+s \in 2 \mathbb{N}}} \frac{\widetilde{\mathcal{R}}_{\Delta, r s}^{f}\left[\begin{array}{c}
-\Delta_{3}-\Delta_{2} \\
\Delta_{4}-\Delta_{1}
\end{array}\right]}{c-c_{r s}(\Delta)}
$$

where $c_{r s}(\Delta)$ have the form $(3.6)$.

The function $\mathrm{h}_{c, \Delta}^{f}\left[\begin{array}{cc}-\Delta_{3}-\Delta_{2} \\ \Delta_{4} & \Delta_{1}\end{array}\right]$ can be determined from the asymptotic behavior of $F_{c, \Delta}^{f}$ for large $\Delta$, while for $\mathrm{f}_{\Delta}^{f}\left[\begin{array}{cc}-\Delta_{3}-\Delta_{2} \\ \Delta_{4} & \Delta_{1}\end{array}\right]$ we simply have

$$
\mathrm{f}_{\Delta}^{f}\left[\begin{array}{cc}
-\Delta_{3} & \Delta_{2} \\
\Delta_{4} & \Delta_{1}
\end{array}\right]=\lim _{c \rightarrow \infty} F_{c, \Delta}^{f}\left[\begin{array}{cc}
-\Delta_{3} & \Delta_{2} \\
\Delta_{4} & \Delta_{1}
\end{array}\right]
$$

Since $\Delta_{r s}\left(c_{r s}(\Delta)\right)=\Delta$, the residue at $\Delta_{r s}(c)$ in (6.1) and at $c_{r s}(\Delta)$ in (6.2) are related by

$$
\begin{aligned}
\widetilde{\mathcal{R}}_{\Delta, r s}^{f}\left[\begin{array}{cc}
-\Delta_{3}-\Delta_{2} \\
\Delta_{4}-\Delta_{1}
\end{array}\right] & =-\frac{\partial c_{r s}(\Delta)}{\partial \Delta} \mathcal{R}_{c_{r s}(\Delta), r s}^{f}\left[\begin{array}{cc}
-\Delta_{3} \Delta_{2} \\
\Delta_{4} \Delta_{1}
\end{array}\right] \\
\frac{\partial c_{r s}(\Delta)}{\partial \Delta} & =\frac{8 c_{r s}(\Delta)-12}{\left(r^{2}-1\right) \beta_{r s}^{4}(\Delta)-\left(s^{2}-1\right)}
\end{aligned}
$$

We shall start with calculating the residue at $\Delta_{r s}$. The corresponding pole arises due to the existence of a singular vector $\chi_{r s} \in \mathcal{V}_{\Delta_{r s}}^{\frac{r s}{2}}$. The rank of the zero of the Kac determinant at $\Delta=\Delta_{r s}$ shows that in a generic case (i.e. when the supermodule $\mathcal{V}_{\Delta_{r s}+\frac{r s}{2}}$ is irreducible) all the null vectors in $\mathcal{V}_{\Delta_{r s}}^{f}$ are descendants of $\chi_{r s}$.

Let $\chi_{r s}^{K M}$ be the coefficients of $\chi_{r s}$ in the basis $S_{-K} L_{-M} \nu_{\Delta_{r s}}$,

$$
\chi_{r s}=\sum_{K, M} \chi_{r s}^{K M} S_{-K} L_{-M} \nu_{\Delta_{r s}} .
$$

We normalize $\chi_{r s}$ such that for $r s \in 2 \mathbb{N}$ the coefficient at $\left(L_{-1}\right)^{\frac{r s}{2}} \nu_{\Delta_{r s}}$, and for $r s \in 2 \mathbb{N}-1$ the coefficient at $S_{-\frac{1}{2}}\left(L_{-1}\right)^{\frac{r s-1}{2}} \nu_{\Delta_{r s}}$, is equal 1 .

For $f>\frac{r s}{2}$ consider vectors of the form

$$
S_{-K} L_{-M} \chi_{r s}^{\Delta} \in \mathcal{V}_{\Delta}^{f}, \quad|K|+|M|=f-\frac{r s}{2},
$$


where

$$
\chi_{r s}^{\Delta}=\sum_{K, M} \chi_{r s}^{K M} S_{-K} L_{-M} \nu_{\Delta}
$$

so that $\chi_{r s}=\lim _{\Delta \rightarrow \Delta_{r s}} \chi_{r s}^{\Delta}$. The set of these vectors can be always extended to a full basis in $\mathcal{V}_{\Delta}^{f}$. Working in such a basis and using the properties of the Gram matrix $B_{c, \Delta}^{f}$ and its inverse one gets

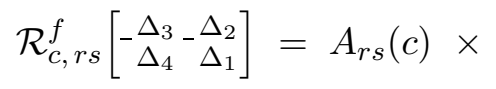

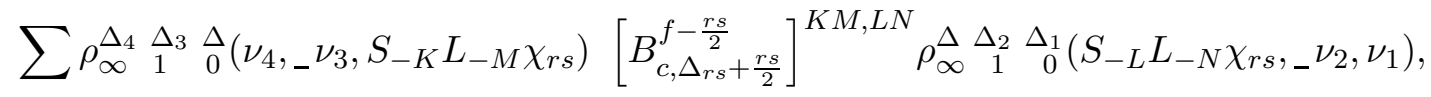

with

$$
A_{r s}(c)=\lim _{\Delta \rightarrow \Delta_{r s}}\left(\frac{\left\langle\chi_{r s}^{\Delta} \mid \chi_{r s}^{\Delta}\right\rangle}{\Delta-\Delta_{r s}(c)}\right)^{-1} .
$$

The factorization (4.16) and the reflection properties (4.14), (4.15) of the form $\rho$ give:

$$
\begin{aligned}
& \mathcal{R}_{c, r s}^{f}\left[\begin{array}{cc}
-\Delta_{3}-\Delta_{2} \\
\Delta_{4} & \Delta_{1}
\end{array}\right]=A_{r s}(c) S_{r s}\left(\Delta_{3} \Delta_{3}\right)
\end{aligned}
$$

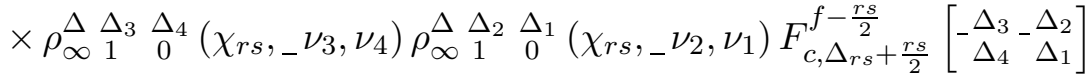

for $f-\frac{r s}{2} \in \mathbb{N} \cup\{0\}$, and

$$
\begin{aligned}
\mathcal{R}_{c, r s}^{f}\left[\begin{array}{rr}
-\Delta_{3}-\Delta_{2} \\
\Delta_{4} & \Delta_{1}
\end{array}\right] & =A_{r s}(c) S_{r s}\left(\Delta_{-} \Delta_{3}\right) \\
\rho_{\infty}^{\Delta} \Delta_{3} & \Delta_{4}
\end{aligned}
$$

for $f-\frac{r s}{2} \in \mathbb{N}-\frac{1}{2}$, where $\widetilde{\nu}=* \nu, \widetilde{* \nu}=\nu$, and

$$
S_{r s}(\Delta)=1 \quad, \quad S_{r s}(* \Delta)=(-1)^{r s} .
$$

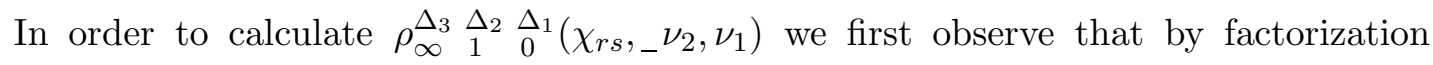
(4.16):

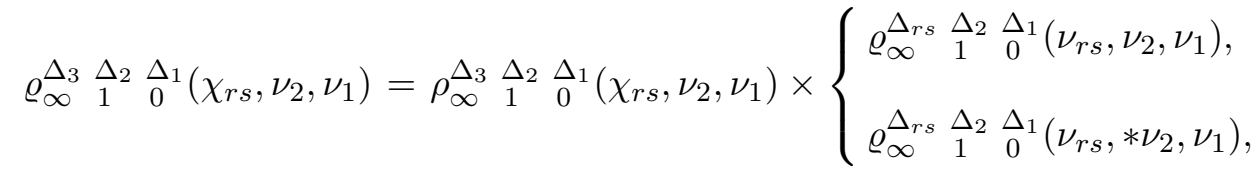

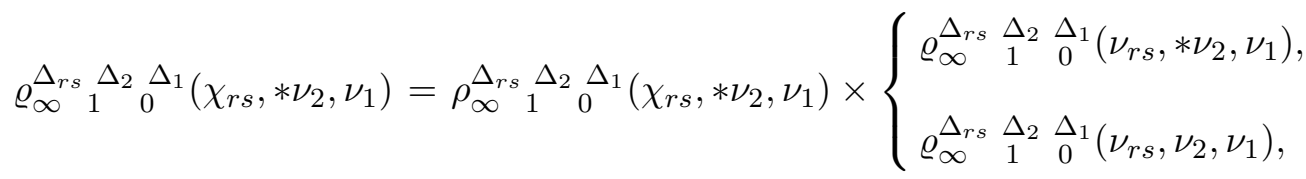

where $r s \in 2 \mathbb{N}$ in the upper lines, $r s \in 2 \mathbb{N}+1$ in the lower lines, and $\nu_{r s} \equiv \nu_{\Delta_{r s}(c)}$.

The Feigin-Fuchs construction allows to represent $\varrho_{\infty}^{\Delta_{r s}}{\underset{1}{1}}_{2}^{\Delta_{1}} \Delta_{1}\left(\nu_{r s}, \nu_{2}, \nu_{1}\right)$ as the left (chiral) part of the three point "screened" correlator [10]. It is non-zero provided the weights $\Delta_{i}=-\frac{1}{8}\left(\beta-\frac{1}{\beta}\right)^{2}+\frac{\alpha_{i}^{2}}{8}$ satisfy the even fusion rule:

$$
\alpha_{2} \pm \alpha_{1}=(1-r+2 k) \beta-(1-s+2 l) \frac{1}{\beta}, \quad k+l \in 2 \mathbb{N} \cup\{0\}
$$


where $k, l$ are integers in the range $0 \leq k \leq r-1,0 \leq l \leq s-1$. Similarly, one gets

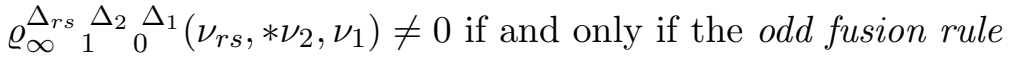

$$
\alpha_{2} \pm \alpha_{1}=(1-r+2 k) \beta-(1-s+2 l) \frac{1}{\beta}, \quad k+l \in 2 \mathbb{N}-1,
$$

is satisfied. Since the definition (4.1) implies that for the null vector $\chi_{r s}$

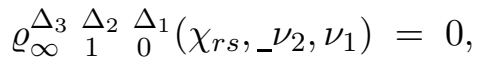

we conclude from (6.10) and (6.11) that also for the normalized form

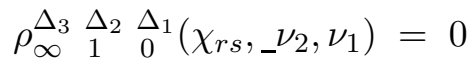

if the appropriate fusion rule is satisfied.

Let us define the "fusion polynomials"

$$
P_{c}^{r s}\left[\begin{array}{c}
\Delta_{2} \\
\Delta_{1}
\end{array}\right]=\prod_{p=1-r}^{r-1} \prod_{q=1-s}^{s-1}\left(\frac{\alpha_{2}-\alpha_{1}+p \beta-q \beta^{-1}}{2 \sqrt{2}}\right)\left(\frac{\alpha_{2}+\alpha_{1}+p \beta-q \beta^{-1}}{2 \sqrt{2}}\right)
$$

where $p+q-(r+s) \in 4 \mathbb{Z}+2(p$ and $q$ are related to the previously used variables through $p=r-1-2 k, q=s-1-2 l)$ and

$$
P_{c}^{r s}\left[\begin{array}{r}
* \Delta_{2} \\
\Delta_{1}
\end{array}\right]=\prod_{p=1-r}^{r-1} \prod_{q=1-s}^{s-1}\left(\frac{\alpha_{2}-\alpha_{1}+p \beta-q \beta^{-1}}{2 \sqrt{2}}\right)\left(\frac{\alpha_{2}+\alpha_{1}+p \beta-q \beta^{-1}}{2 \sqrt{2}}\right)
$$

with $p+q-(r+s) \in 4 \mathbb{Z}$. The following properties can be easily obtained by simple combinatorics:

1. $P_{c}^{r s}\left[\begin{array}{l}\Delta_{2} \\ \Delta_{1}\end{array}\right]$ vanishes if the even fusion rule is satisfied and $P_{c}^{r s}\left[\begin{array}{c}* \Delta_{2} \\ \Delta_{1}\end{array}\right]$ if odd fusion rule holds;

2. $P_{c}^{r s}\left[\begin{array}{l}\Delta_{2} \\ \Delta_{1}\end{array}\right]$ is a polynomial of degree $\left[\frac{r s+1}{2}\right]$ in the variable $\Delta_{2}-\Delta_{1}$ and of degree $\left[\frac{r s+1}{4}\right]$ in $\Delta_{2}+\Delta_{1}$ and $P_{c}^{r s}\left[\begin{array}{c}* \Delta_{2} \\ \Delta_{1}\end{array}\right]$ is a polynomial of degree $\left[\frac{r s}{2}\right]$ in the variable $\Delta_{2}-\Delta_{1}$ and of degree $\left[\frac{r s}{4}\right]$ in $\Delta_{2}+\Delta_{1}$;

3. coefficients of highest powers of $\Delta_{2}-\Delta_{1}$ in both polynomials are equal 1 .

The properties above uniquely determine polynomials $P_{c}^{r s}$. On the other hand, it follows from (6.12), (4.12), (4.13) and the normalization of $\chi_{r s}$ that with an appropriate choice of the second argument the same properties are satisfied by $\rho_{\infty}^{\Delta} \Delta_{3}^{\Delta} \underset{0}{\Delta_{4}}\left(\chi_{r s}, \nu_{2}, \nu_{1}\right)$ as well. This implies the equalities:

$$
\begin{aligned}
& \rho_{\infty 1}^{\Delta \Delta_{3}} \underset{0}{\underset{\Delta_{4}}{0}}\left(\chi_{r s}, \nu_{2}, \nu_{1}\right)= \begin{cases}P_{c}^{r s}\left[\begin{array}{l}
\Delta_{2} \\
\Delta_{1}
\end{array}\right] & \text { for } \frac{r s}{2} \in \mathbb{N}, \\
P_{c}^{r s}\left[\begin{array}{c}
* \Delta_{2} \\
\Delta_{1}
\end{array}\right] & \text { for } \frac{r s}{2} \in \mathbb{N}-\frac{1}{2}\end{cases} \\
& \rho_{\infty}^{\Delta} \Delta_{3} \underset{\Delta_{4}}{\Delta_{4}}\left(\chi_{r s}, * \nu_{2}, \nu_{1}\right)= \begin{cases}P_{c}^{r s}\left[\begin{array}{c}
* \Delta_{2} \\
\Delta_{1}
\end{array}\right] & \text { for } \frac{r s}{2} \in \mathbb{N} \\
P_{c}^{r s}\left[\begin{array}{c}
\Delta_{2} \\
\Delta_{1}
\end{array}\right] & \text { for } \frac{r s}{2} \in \mathbb{N}-\frac{1}{2}\end{cases}
\end{aligned}
$$


In order to complete our derivation of $\mathcal{R}_{c, r s}^{f}\left[\begin{array}{cc}-\Delta_{3} & \Delta_{2} \\ \Delta_{4} & \Delta_{1}\end{array}\right]$ we thus only need the coefficients $A_{r s}(c)$. Fortunately the r.h.s. of $(6.6)$ can be easily calculated using equations $(43)-(46)$ of [11] (note that in the present case we calculate the residue at $\Delta_{r s}$ rather than at $\alpha_{r s}$ ). In our notation the result reads:

$$
A_{r s}(c)=\frac{1}{2}(-1)^{r s-1} \prod_{p=1-r}^{r} \prod_{q=1-s}^{s}\left(\frac{1}{\sqrt{2}}\left(p \beta-\frac{q}{\beta}\right)\right)^{-1}, \quad p+q \in 2 \mathbb{Z},(p, q) \neq(0,0),(r, s) .
$$

Our final formulae for the residue at $\Delta_{r s}$ take the form:

$$
\mathcal{R}_{c, r s}^{m}\left[\begin{array}{cc}
-\Delta_{3} & -\Delta_{2} \\
\Delta_{4} & \Delta_{1}
\end{array}\right]=A_{r s}(c) S_{r s}\left({ }_{-} \Delta_{3}\right) P_{c}^{r s}\left[\begin{array}{c}
-\Delta_{3} \\
\Delta_{4}
\end{array}\right] P_{c}^{r s}\left[\begin{array}{c}
-\Delta_{2} \\
\Delta_{1}
\end{array}\right] F_{c, \Delta_{r s}+\frac{r s}{2}}^{m-\frac{r s}{2}}\left[\begin{array}{cc}
-\Delta_{3} & \Delta_{2} \\
\Delta_{4} & \Delta_{1}
\end{array}\right]
$$

for $m \in \mathbb{N} \cup\{0\}$ and

$$
\mathcal{R}_{c, r s}^{k}\left[\begin{array}{cc}
-\Delta_{3}-\Delta_{2} \\
\Delta_{4} & \Delta_{1}
\end{array}\right]=A_{r s}(c) S_{r s}\left(\Delta_{3} \Delta_{3}\right) P_{c}^{r s}\left[\begin{array}{c}
\widetilde{\Delta_{3}} \\
\Delta_{4}
\end{array}\right] P_{c}^{r s}\left[\begin{array}{c}
\widetilde{\Delta_{2}} \\
-\Delta_{1}
\end{array}\right] F_{c, \Delta_{r s}+\frac{r s}{2}}^{k-\frac{r s}{2}}\left[\begin{array}{c}
-\Delta_{3}-\Delta_{2} \\
\Delta_{4}-\Delta_{1}
\end{array}\right]
$$

for $k \in \mathbb{N}-\frac{1}{2}$, where the coefficients involved are defined by $(6.9),(6.13),(6.14)$, and (6.16).

Let us now calculate the leading terms in the expansion over the poles in $c$,

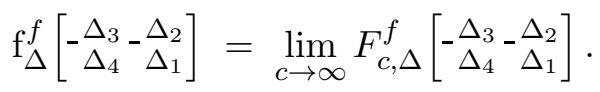

It follows from the $N S$ algebra that the only vectors in $\mathcal{V}_{c, \Delta}^{n}$ with all matrix elements independent of $c$ (an thus finite in the limit $c \rightarrow \infty$ ) are proportional to $L_{-1}^{n}|\Delta\rangle$. For $\mathcal{V}_{c, \Delta}^{n+\frac{1}{2}}$ such vectors are proportional to $S_{-\frac{1}{2}} L_{-1}^{n}|\Delta\rangle$. Consequently, only these states contribute to $(6.19)$. We have:

$$
\begin{aligned}
\left\langle\Delta\left|L_{1}^{n} L_{-1}^{n}\right| \Delta\right\rangle & =n !(2 \Delta)_{n}, \\
\left\langle\Delta\left|L_{1}^{n} S_{\frac{1}{2}} S_{-\frac{1}{2}} L_{-1}^{n}\right| \Delta\right\rangle & =n !(2 \Delta)_{n+1},
\end{aligned}
$$

where

$$
(a)_{n}=\frac{\Gamma(a+n)}{\Gamma(n)}
$$

is the Pochhammer symbol.

In the limit $c \rightarrow \infty$ the corresponding elements of the inverse Gram matrix are just inverses of the matrix elements (6.20). Furthermore, from (4.12) and (4.13):

$$
\begin{aligned}
& \left.\rho_{\infty}^{\Delta} \begin{array}{c}
\Delta_{2} \\
\Delta_{1}
\end{array} \mathrm{\Delta}_{-1}^{n} \nu, \nu_{2}, \nu_{1}\right)=\left(\Delta+\Delta_{2}-\Delta_{1}\right)_{n}, \\
& \rho_{\infty}^{\Delta \Delta_{2} \Delta_{1}}\left(S_{-\frac{1}{2}} \mathrm{~L}_{-1}^{n} \nu, \nu_{2}, \nu_{1}\right)=\left(\Delta+\Delta_{2}-\Delta_{1}+\frac{1}{2}\right)_{n}, \\
& \rho_{\infty}^{\Delta \Delta_{2}} \underset{1}{\Delta_{1}} \Delta_{1}\left(\mathrm{~L}_{-1}^{n} \nu, * \nu_{2}, \nu_{1}\right)=\left(\Delta+\Delta_{2}-\Delta_{1}+\frac{1}{2}\right)_{n}, \\
& \rho_{\infty}^{\Delta} \underset{1}{\Delta} \underset{2}{\Delta_{2}} \underset{1}{\Delta}\left(S_{-\frac{1}{2}} \mathrm{~L}_{-1}^{n} \nu, * \nu_{2}, \nu_{1}\right)=-\rho_{0}^{\Delta_{1}} \underset{1}{\Delta_{2} \Delta}\left(\nu_{1}, * \nu_{2}, S_{-\frac{1}{2}} \mathrm{~L}_{-1}^{n} \nu\right)=\left(\Delta+\Delta_{2}-\Delta_{1}\right)_{n+1} .
\end{aligned}
$$


We thus get:

$$
\begin{aligned}
\mathrm{f}_{\Delta}^{n}\left[\begin{array}{ll}
\Delta_{3} & \Delta_{2} \\
\Delta_{4} & \Delta_{1}
\end{array}\right] & =\frac{1}{n !} \frac{\left(\Delta+\Delta_{3}-\Delta_{4}\right)_{n}\left(\Delta+\Delta_{2}-\Delta_{1}\right)_{n}}{(2 \Delta)_{n}}, \\
\mathrm{f}_{\Delta}^{n+\frac{1}{2}}\left[\begin{array}{ll}
\Delta_{3} & \Delta_{2} \\
\Delta_{4} & \Delta_{1}
\end{array}\right] & =\frac{1}{n !} \frac{\left(\Delta+\Delta_{3}-\Delta_{4}+\frac{1}{2}\right)_{n}\left(\Delta+\Delta_{2}-\Delta_{1}+\frac{1}{2}\right)_{n}}{(2 \Delta)_{n+1}}, \\
\mathrm{f}_{\Delta}^{n}\left[\begin{array}{cc}
* \Delta_{3} & * \Delta_{2} \\
\Delta_{4} & \Delta_{1}
\end{array}\right] & =\frac{1}{n !} \frac{\left(\Delta+\Delta_{3}-\Delta_{4}+\frac{1}{2}\right)_{n}\left(\Delta+\Delta_{2}-\Delta_{1}+\frac{1}{2}\right)_{n}}{(2 \Delta)_{n}}, \\
\mathrm{f}_{\Delta}^{n+\frac{1}{2}}\left[\begin{array}{rr}
* \Delta_{3} & * \Delta_{2} \\
\Delta_{4} & \Delta_{1}
\end{array}\right] & =-\frac{1}{n !} \frac{\left(\Delta+\Delta_{3}-\Delta_{4}\right)_{n+1}\left(\Delta+\Delta_{2}-\Delta_{1}\right)_{n+1}}{(2 \Delta)_{n+1}}
\end{aligned}
$$

and so on.

Substituting $(6.3),(6.17),(6.18)$ and $(6.21)$ into $(6.2)$, and introducing simplified notation

$$
\tilde{A}_{r s}(\Delta)=-\frac{\partial c_{r s}(\Delta)}{\partial \Delta} A_{r s}\left(c_{r s}(\Delta)\right), \quad P_{\Delta}^{r s}\left[\begin{array}{c}
-\Delta_{a} \\
\Delta_{b}
\end{array}\right]=P_{c_{r s}(\Delta)}^{r s}\left[\begin{array}{c}
-\Delta_{a} \\
\Delta_{b}
\end{array}\right]
$$

one finally gets the recursion relations for the coefficients in the $x$-expansion of the NS superconformal blocks

$$
\begin{aligned}
F_{c, \Delta}^{m}\left[\begin{array}{rr}
-\Delta_{3} & \Delta_{2} \\
\Delta_{4} & \Delta_{1}
\end{array}\right] & =\mathrm{f}_{\Delta}^{m}\left[\begin{array}{rr}
-\Delta_{3} & \Delta_{2} \\
\Delta_{4} & \Delta_{1}
\end{array}\right] \\
& +\sum_{\substack{1<r s \leq 2 f, 1<r \\
r+s \in 2 \mathbb{N}}} \frac{\tilde{A}_{r s}(\Delta)}{c-c_{r s}(\Delta)} P_{\Delta}^{r s}\left[\begin{array}{r}
-\Delta_{3} \\
\Delta_{4}
\end{array}\right] P_{\Delta}^{r s}\left[\begin{array}{c}
-\Delta_{2} \\
\Delta_{1}
\end{array}\right] F_{c_{r s}, \Delta+\frac{r s}{2}}^{m-\frac{r s}{2}}\left[\begin{array}{c}
-\Delta_{3} \\
\Delta_{4}-\Delta_{2} \\
\Delta_{1}
\end{array}\right],
\end{aligned}
$$

where $m \in \mathbb{N}$ and

$$
\begin{aligned}
F_{c, \Delta}^{k}\left[\begin{array}{rr}
-\Delta_{3} & -\Delta_{2} \\
\Delta_{4} & \Delta_{1}
\end{array}\right] & =\mathrm{f}_{\Delta}^{k}\left[\begin{array}{rr}
-\Delta_{3} & -\Delta_{2} \\
\Delta_{4} & \Delta_{1}
\end{array}\right] \\
& \pm \sum_{\substack{1<r s \leq 2 f, 1<r \\
r+s \in 2 \mathbb{N}}} \frac{\tilde{A}_{r s}(\Delta)}{c-c_{r s}(\Delta)} P_{\Delta}^{r s}\left[\begin{array}{r}
-\Delta_{3} \\
\Delta_{4}
\end{array}\right] P_{\Delta}^{r s}\left[\begin{array}{c}
\widetilde{\Delta_{2}} \\
\Delta_{1}
\end{array}\right] F_{c_{r s}, \Delta+\frac{r s}{2}}^{k-\frac{r s}{2}}\left[\begin{array}{c}
-\Delta_{3}-\Delta_{2} \\
\Delta_{4}-\Delta_{1}
\end{array}\right]
\end{aligned}
$$

where $k \in \mathbb{N}-\frac{1}{2}$, and $+/-$ in the second line correspond to $\Delta_{3} / * \Delta_{3}$, respectively. Let us note that for each type of the NS superconformal block one gets independent recursion formulae mixing coefficients of the even and the odd blocks.

\section{Acknowledgements}

The work of L.H and Z.J. was partially supported by the Polish State Research Committee (KBN) grant no. 1 P03B 02528.

The research of L.H. is supported by the Alexander von Humboldt Foundation scholarship. L.H. would like to thank Alexander Belavin for sharing with him his interest in the topics discussed in the present work and numerous helpful discussions at the early stage of the project.

P.S. is grateful to the faculty of the Institute of Theoretical Physics, University of Wrocław, for the hospitality. 


\section{Appendix A. Explicit calculations at $f=\frac{3}{2}, 2$, and $\frac{5}{2}$}

In this appendix we present some explicit calculation for the first few coefficients of "symmetric" conformal blocks. Our purpose is to illustrate how the presented method works and to provide a simple check of the formulae derived.

At $f=\frac{1}{2}$ and $f=1$ there are no $c$ poles in the Gram matrix and the coefficients of the blocks are fully determined by the asymptotic terms (6.21).

For $f=\frac{3}{2}$ there is a pole at

$$
c=c_{31}(\Delta)=\frac{3 \Delta(3-2 \Delta)}{2 \Delta+1} \Rightarrow-\frac{\partial c_{31}(\Delta)}{\partial \Delta}=3 \frac{(2 \Delta-1)(2 \Delta+3)}{(2 \Delta+1)^{2}} .
$$

We have:

$$
\begin{gathered}
F_{c, \Delta}^{\frac{3}{2}}\left[\begin{array}{cc}
* \Delta_{3} & * \Delta_{2} \\
\Delta_{4} & \Delta_{1}
\end{array}\right]=\mathrm{f}_{\Delta}^{\frac{3}{2}}\left[\begin{array}{cc}
* \Delta_{3} & * \Delta_{2} \\
\Delta_{4} & \Delta_{1}
\end{array}\right]-\frac{\tilde{A}_{31}(\Delta)}{c-c_{31}} P_{\Delta}^{31}\left[\begin{array}{c}
\Delta_{2} \\
\Delta_{1}
\end{array}\right] P_{\Delta}^{31}\left[\begin{array}{c}
\Delta_{3} \\
\Delta_{4}
\end{array}\right] \\
F_{c, \Delta}^{\frac{3}{2}}\left[\begin{array}{ll}
\Delta_{3} & \Delta_{2} \\
\Delta_{4} & \Delta_{1}
\end{array}\right]=\mathrm{f}_{\Delta}^{\frac{3}{2}}\left[\begin{array}{cc}
\Delta_{3} & \Delta_{2} \\
\Delta_{4} & \Delta_{1}
\end{array}\right]+\frac{\tilde{A}_{31}(\Delta)}{c-c_{31}} P_{\Delta}^{31}\left[\begin{array}{c}
* \Delta_{2} \\
\Delta_{1}
\end{array}\right] P_{\Delta}^{31}\left[\begin{array}{c}
* \Delta_{3} \\
\Delta_{4}
\end{array}\right],
\end{gathered}
$$

with (from 6.13), (6.14) and (6.22))

$$
\begin{aligned}
P_{\Delta}^{31}\left[\begin{array}{c}
\Delta_{2} \\
\Delta_{1}
\end{array}\right] & =\frac{1}{2}\left(2\left(\Delta_{2}-\Delta_{1}\right)^{2}-(2 \Delta+1)\left(\Delta_{2}+\Delta_{1}\right)+\Delta\right), \\
P_{\Delta}^{31}\left[\begin{array}{r}
* \Delta_{2} \\
\Delta_{1}
\end{array}\right] & =\left(\Delta_{2}-\Delta_{1}\right)
\end{aligned}
$$

and (from $(\underline{6.16})$ and $(\underline{6.22})$

$$
\tilde{A}_{31}(\Delta)=\frac{6}{(2 \Delta+1)^{2}}
$$

These formulae are easily checked using the definitions from the section 5 and an explicit expression for the Gram matrix, which in the basis $\left\{S_{-\frac{1}{2}} L_{-1}|\Delta\rangle, S_{-\frac{3}{2}}|\Delta\rangle\right\}$ reads:

$$
\left[B_{c, \Delta}^{\frac{3}{2}}\right]=\left(\begin{array}{cc}
2 \Delta(2 \Delta+1) & 4 \Delta \\
4 \Delta & 2 \Delta+\frac{2}{3} c
\end{array}\right) .
$$

For $f=2$ we have poles at $c=c_{31}(\Delta)$ and

$$
c=c_{22}(\Delta)=\frac{3}{2}-8 \Delta \text {. }
$$

With

$$
\begin{aligned}
P_{\Delta}^{22}\left[\begin{array}{c}
\Delta_{2} \\
\Delta_{1}
\end{array}\right] & =\frac{1}{3}\left(3\left(\Delta_{2}-\Delta_{1}\right)^{2}-2 \Delta\left(\Delta_{2}+\Delta_{1}\right)-\Delta^{2}\right) \\
P_{\Delta}^{22}\left[\begin{array}{c}
* \Delta_{2} \\
\Delta_{1}
\end{array}\right] & =\frac{1}{12}\left(12\left(\Delta_{2}-\Delta_{1}\right)^{2}-4(2 \Delta+3)\left(\Delta_{2}+\Delta_{1}\right)-(2 \Delta+3)(2 \Delta-1)\right)
\end{aligned}
$$

and

$$
\tilde{A}_{22}(\Delta)=\frac{9}{4 \Delta(2 \Delta+3)},
$$


we have:

$$
\begin{aligned}
& F_{c, \Delta}^{2}\left[\begin{array}{ll}
\Delta_{3} & \Delta_{2} \\
\Delta_{4} & \Delta_{1}
\end{array}\right]=\mathrm{f}_{\Delta}^{2}\left[\begin{array}{ll}
\Delta_{3} & \Delta_{2} \\
\Delta_{4} & \Delta_{1}
\end{array}\right]+\frac{\tilde{A}_{22}(\Delta)}{c-c_{22}} P_{\Delta}^{22}\left[\begin{array}{l}
\Delta_{2} \\
\Delta_{1}
\end{array}\right] P_{\Delta}^{22}\left[\begin{array}{l}
\Delta_{3} \\
\Delta_{4}
\end{array}\right] \\
& +\frac{\tilde{A}_{31}(\Delta)}{c-c_{31}} P_{\Delta}^{31}\left[\begin{array}{l}
\Delta_{2} \\
\Delta_{1}
\end{array}\right] P_{\Delta}^{31}\left[\begin{array}{l}
\Delta_{3} \\
\Delta_{4}
\end{array}\right] \mathrm{f}_{\Delta+\frac{3}{2}}^{\frac{1}{2}}\left[\begin{array}{ll}
\Delta_{3} & \Delta_{2} \\
\Delta_{4} & \Delta_{1}
\end{array}\right], \\
& F_{c, \Delta}^{2}\left[\begin{array}{cc}
* \Delta_{3} & * \Delta_{2} \\
\Delta_{4} & \Delta_{1}
\end{array}\right]=\mathrm{f}_{\Delta}^{2}\left[\begin{array}{cc}
* \Delta_{3} & * \Delta_{2} \\
\Delta_{4} & \Delta_{1}
\end{array}\right]+\frac{\tilde{A}_{22}(\Delta)}{c-c_{22}} P_{\Delta}^{22}\left[\begin{array}{c}
* \Delta_{2} \\
\Delta_{1}
\end{array}\right] P_{\Delta}^{22}\left[\begin{array}{c}
* \Delta_{3} \\
\Delta_{4}
\end{array}\right] \\
& +\frac{\tilde{A}_{31}(\Delta)}{c-c_{31}} P_{\Delta}^{31}\left[\begin{array}{r}
* \Delta_{2} \\
\Delta_{1}
\end{array}\right] P_{\Delta}^{31}\left[\begin{array}{r}
* \Delta_{3} \\
\Delta_{4}
\end{array}\right] \mathrm{f}_{\Delta+\frac{3}{2}}^{\frac{1}{2}}\left[\begin{array}{cc}
* \Delta_{3} & * \Delta_{2} \\
\Delta_{4} & \Delta_{1}
\end{array}\right]
\end{aligned}
$$

what can be checked noting that in the basis $\left\{L_{-1} L_{-1}|\Delta\rangle, L_{-2}|\Delta\rangle, S_{-\frac{1}{2}} S_{-\frac{3}{2}}|\Delta\rangle\right\}$ the Gram matrix reads

$$
\left[B_{c, \Delta}^{2}\right]=\left(\begin{array}{ccc}
4 \Delta(2 \Delta+1) & 6 \Delta & 8 \Delta \\
6 \Delta & 4 \Delta+\frac{c}{2} & 3 \Delta+c \\
8 \Delta & 3 \Delta+c & 4 \Delta\left(\Delta+\frac{c}{3}\right)+2(c-\Delta)
\end{array}\right) .
$$

Finally, for $f=\frac{5}{2}$ there appears a "new" pole at

$$
c=c_{51}(\Delta)=\frac{(2 \Delta-1)(5-\Delta)}{2 \Delta+2} \Rightarrow \quad-\frac{\partial c_{51}(\Delta)}{\partial \Delta}=\frac{(\Delta-2)(\Delta+4)}{(\Delta+1)^{2}} .
$$

The fusion polynomials read

$$
\begin{aligned}
P_{\Delta}^{51}\left[\begin{array}{c}
\Delta_{2} \\
\Delta_{1}
\end{array}\right] & =\frac{1}{3}\left(\Delta_{2}-\Delta_{1}\right)\left(3\left(\Delta_{2}-\Delta_{1}\right)^{2}-4(\Delta+1)\left(\Delta_{2}+\Delta_{1}\right)+\Delta(\Delta+4)\right), \\
P_{\Delta}^{51}\left[\begin{array}{c}
* \Delta_{2} \\
\Delta_{1}
\end{array}\right] & =\frac{1}{12}\left(12\left(\Delta_{2}-\Delta_{1}\right)^{2}-4(\Delta+1)\left(\Delta_{2}+\Delta_{1}\right)+2 \Delta-1\right),
\end{aligned}
$$

and with

$$
\tilde{A}_{51}(\Delta)=\frac{9}{8 \Delta(\Delta+2)(\Delta+1)^{2}}
$$

we have:

$$
\begin{aligned}
& F_{c, \Delta}^{\frac{5}{2}}\left[\begin{array}{cc}
* \Delta_{3} & * \Delta_{2} \\
\Delta_{4} & \Delta_{1}
\end{array}\right]=\mathrm{f}_{\Delta}^{\frac{5}{2}}\left[\begin{array}{cc}
* \Delta_{3} & * \Delta_{2} \\
\Delta_{4} & \Delta_{1}
\end{array}\right]-\frac{\tilde{A}_{51}(\Delta)}{c-c_{51}} P_{\Delta}^{51}\left[\begin{array}{c}
\Delta_{2} \\
\Delta_{1}
\end{array}\right] P_{\Delta}^{51}\left[\begin{array}{c}
\Delta_{3} \\
\Delta_{4}
\end{array}\right] \\
& +\frac{\tilde{A}_{22}(\Delta)}{c-c_{22}} P_{\Delta}^{22}\left[\begin{array}{c}
\Delta_{2} \\
\Delta_{1}
\end{array}\right] P_{\Delta}^{22}\left[\begin{array}{l}
\Delta_{3} \\
\Delta_{4}
\end{array}\right] \mathrm{f}_{\Delta+2}^{\frac{1}{2}}\left[\begin{array}{cc}
* \Delta_{3} & * \Delta_{2} \\
\Delta_{4} & \Delta_{1}
\end{array}\right] \\
& -\frac{\tilde{A}_{31}(\Delta)}{c-c_{31}} P_{\Delta}^{31}\left[\begin{array}{c}
\Delta_{2} \\
\Delta_{1}
\end{array}\right] P_{\Delta}^{31}\left[\begin{array}{c}
\Delta_{3} \\
\Delta_{4}
\end{array}\right] \mathrm{f}_{\Delta+\frac{3}{2}}^{1}\left[\begin{array}{cc}
* \Delta_{3} & * \Delta_{2} \\
\Delta_{4} & \Delta_{1}
\end{array}\right] \text {, } \\
& F_{c, \Delta}^{\frac{5}{2}}\left[\begin{array}{ll}
\Delta_{3} & \Delta_{2} \\
\Delta_{4} & \Delta_{1}
\end{array}\right]=\mathrm{f}_{\Delta}^{\frac{5}{2}}\left[\begin{array}{cc}
\Delta_{3} & \Delta_{2} \\
\Delta_{4} & \Delta_{1}
\end{array}\right]+\frac{\tilde{A}_{51}(\Delta)}{c-c_{51}} P_{\Delta}^{51}\left[\begin{array}{c}
* \Delta_{2} \\
\Delta_{1}
\end{array}\right] P_{\Delta}^{51}\left[\begin{array}{c}
* \Delta_{3} \\
\Delta_{4}
\end{array}\right] \\
& +\frac{\tilde{A}_{22}(\Delta)}{c-c_{22}} P_{\Delta}^{22}\left[\begin{array}{c}
* \Delta_{2} \\
\Delta_{1}
\end{array}\right] P_{\Delta}^{22}\left[\begin{array}{c}
* \Delta_{3} \\
\Delta_{4}
\end{array}\right] \mathrm{f}_{\Delta+2}^{\frac{1}{2}}\left[\begin{array}{ll}
\Delta_{3} & \Delta_{2} \\
\Delta_{4} & \Delta_{1}
\end{array}\right] \\
& +\frac{\tilde{A}_{31}(\Delta)}{c-c_{31}} P_{\Delta}^{31}\left[\begin{array}{r}
* \Delta_{2} \\
\Delta_{1}
\end{array}\right] P_{\Delta}^{31}\left[\begin{array}{c}
* \Delta_{3} \\
\Delta_{4}
\end{array}\right] \mathrm{f}_{\Delta+\frac{3}{2}}^{1}\left[\begin{array}{ll}
\Delta_{3} & \Delta_{2} \\
\Delta_{4} & \Delta_{1}
\end{array}\right] .
\end{aligned}
$$


The results above fully agree with the direct calculation in which the Gram matrix, with the form

$$
\left[B_{c, \Delta}^{\frac{5}{2}}\right]=\left(\begin{array}{cccc}
8 \Delta(\Delta+1)(2 \Delta+1) & 12 \Delta(\Delta+1) & 4 \Delta(4 \Delta+1) & 12 \Delta \\
12 \Delta(\Delta+1) & (\Delta+2)(8 \Delta+c)-\frac{3}{2}(3 \Delta+c) & 7 \Delta & 8 \Delta+c \\
4 \Delta(4 \Delta+1) & 7 \Delta & \frac{4}{3} \Delta(3 \Delta+3+c) & 4 \Delta \\
12 \Delta & 8 \Delta+c & 4 \Delta & 2 \Delta+2 c
\end{array}\right)
$$

in the basis $\left\{S_{-\frac{1}{2}} L_{-1} L_{-1}|\Delta\rangle, S_{-\frac{1}{2}} L_{-2}|\Delta\rangle, S_{-\frac{3}{2}} L_{-1}|\Delta\rangle, S_{-\frac{5}{2}}|\Delta\rangle\right\}$, is used.

\section{References}

[1] A. A. Belavin, A. M. Polyakov and A. B. Zamolodchikov, Infinite Conformal Symmetry In Two-Dimensional Quantum Field Theory, Nucl. Phys. B 241, 333 (1984).

[2] Al. Zamolodchikov, Conformal Symmetry In Two-Dimensions: An Explicit Recurrence Formula For The Conformal Partial Wave Amplitude, Commun. Math. Phys. 96 (1984) 419.

[3] Al. Zamolodchikov, Two-dimensional conformal symmetry and critical four-spin correlation functions in the Ashkin-Teller model, Sov. Phys. JETP 63 (1986) 1061.

[4] Al. Zamolodchikov, Conformal symmetry in two-dimensional space: recursion representation of conformal block, Theor.Math.Phys. 73 (1987) 1088.

[5] A. B. Zamolodchikov and Al. Zamolodchikov, Structure constants and conformal bootstrap in Liouville field theory, Nucl. Phys. B 477, 577 (1996) [arXiv:hep-th/9506136].

[6] I. Runkel and G. M. T. Watts, A non-rational CFT with $c=1$ as a limit of minimal models, JHEP 0109 (2001) 006 [arXiv:hep-th/0107118].

[7] L. Hadasz, Z. Jaskólski and M. Piạtek, Classical geometry from the quantum Liouville theory, Nucl. Phys. B 724, 529 (2005) [arXiv:hep-th/0504204].

L. Hadasz and Z. Jaskólski, Liouville theory and uniformization of four-punctured sphere, J. Math. Phys. 47, 082304 (2006) [arXiv:hep-th/0604187].

[8] J. Teschner, Liouville theory revisited, Class. Quant. Grav. 18 (2001) R153 [arXiv:hep-th/0104158].

[9] G. W. Moore and N. Seiberg, Polynomial Equations For Rational Conformal Field Theories, Phys. Lett. B 212 (1988) 451.

G. W. Moore and N. Seiberg, Classical And Quantum Conformal Field Theory, Commun. Math. Phys. 123 (1989) 177.

[10] M. A. Bershadsky, V. G. Knizhnik and M. G. Teitelman, Superconformal Symmetry In Two-Dimensions, Phys. Lett. B 151 (1985) 31.

[11] A. A. Belavin and Al. Zamolodchikov, Higher equations of motion in $N=1$ SUSY Liouville field theory, arXiv:hep-th/0610316. 